\title{
Bioconvection in a suspension of phototactic algae
}

\author{
By R. V. VINCENT AND N. A. HILL \\ Department of Applied Mathematical Studies, University of Leeds, Leeds LS2 9JT, UK
}

(Received 27 June 1995 and in revised form 22 July 1996)

In this paper we develop a new generic model for phototaxis in a suspension of microscopic swimming algae. Phototaxis is a directed swimming response dependent upon the light conditions sensed by the microorganisms. Positive phototaxis consists of motions directed toward the source of illumination and negative phototaxis is motion directed away from it. The model also incorporates the effect of shading whereby microorganisms nearer the light source absorb and scatter the light before it reaches those further away. This model of phototaxis and shading is then used to analyse the linear stability of a suspension of phototactic algae, uniformly illuminated from above, that swim in a fluid which is slightly less dense then they are. In the basic state there are no fluid motions and the up and down swimming caused by positive and negative phototaxis is balanced by diffusion, with the result that a horizontal, concentrated layer of algae forms, the vertical position of which depends on the light intensity. This leads to a bulk density stratification with a gravitationally stable layer of fluid overlying an unstable layer. When the resulting density gradient becomes large enough, a Rayleigh-Taylor type instability initiates fluid motions in the lower, unstable region that subsequently penetrate the upper, locally stable region. The behaviour of the suspension is characterized by four parameters: a layer depth parameter $d$, a Rayleigh number $R$, a Schmidt number $S_{c}$, and a sublayer position parameter $C$ that specifies the vertical position of the sublayer in the fluid. Linear stability analysis of the basic state indicates that initial pattern wavelengths and the critical value of $R$ at which the suspension becomes unstable are affected by the position of the sublayer, which is determined by the intensity of the light source, and by the type of boundary at the top of the fluid. Oscillatory modes of disturbance are also predicted for certain parameter ranges, driven by cells swimming vigorously upwards towards darker, concentrated downwelling regions and thus creating lower, positively buoyant regions which oppose the fluid motion in the convection patterns.

\section{Introduction}

Concentrated suspensions of microscopic, swimming algae placed in a shallow dish often spontaneously form dynamic convection patterns (Wager 1911; Loeffer \& Mefferd 1952; Kessler 1985, 1986, 1989). This phenomenon is known as bioconvection (Platt 1961), and may be observed in a large number of different algal species where the cells are denser than water and tend to swim upward on average (Pedley \& Kessler 1992).

Experimental observations indicate that bioconvection patterns are modified by illumination (Wager 1911; Kessler 1985; Roennberg, Colfax \& Hastings 1989; Vincent 
1995). Strong light destroys steady patterns in suspensions of motile algae, or prevents the formation of patterns in well-stirred cultures. Illumination can also affect the size and/or shape of patterns as is very well illustrated by a series of photographs taken by Kessler (1985, figure 12). These show the changes that occur in the convection patterns formed in dim light by the alga Dunaliella tertiolecta that are subsequently subjected to bright illumination from above. Perhaps the most striking effect is the increase in the pattern size, although their structural form changes little. These changes probably arise for two main reasons. Firstly algae obtain energy via the process of photosynthesis, and therefore their swimming trajectories are modified by behavioural responses (called photoresponses) which aim to secure and maintain a position within the environment where the cells can collect an optimal number of photons. Primary amongst the photoresponses is phototaxis, which is defined as the directed movement of an organism with respect to the direction of light. It occurs in two general forms: positive motions, directed toward the light source, and negative motion, directed away from the light source (Häder 1987a; Colombetti \& Petracchi 1989). Typically motile algae will swim toward weak sources of light and away from strong sources of light. The second source of pattern change arises from suspension shading (Wager 1911; Kessler 1989). Shading is simply the process by which algae closer to the light source absorb and scatter the wavelengths of light necessary for photosynthesis before it reaches those further away, thereby modifying the collective behaviour observed through the resulting competition for the available light.

The aim of this paper is to formulate a generic theoretical model of phototaxis coupled with suspension shading, and to use it to analyse the stability of a suspension of phototactic algae in a fluid medium which is slightly less dense then they are and which is subject to uniform, overhead illumination.

Previous models of bioconvection in suspensions of motile microorganisms have centred around up-swimming (negatively gravitactic or geotactic) cells (Childress, Levandowsky \& Spiegel 1975, hereinafter referred to as CLS), gyrotactic cells (Kessler 1985; Pedley, Hill \& Kessler 1988; Hill, Pedley \& Kessler 1989; Pedley \& Kessler 1990) and chemotactic cells (Kessler 1989; Hillesdon 1994; Hillesdon, Pedley \& Kessler 1995). CLS examined the stability of a finite-depth layer of up-swimming organisms. They found that the wavelength of the most unstable mode was always infinite. Hill et al. (1989, hereafter referred to as HPK) repeated this analysis for a suspension of gyrotactic algae, using the model for gyrotaxis previously developed by Pedley et al. (1988), and found that the critical initial wavelength was always finite. Their model also predicted that oscillatory modes of instability could occur for particular parameter ranges when the upper surface was rigid. Pedley (1988) and Pedley \& Kessler (1990) examined the initial stages of gyrotactic instabilities in suspensions of algae that had infinite depth. Hillesdon (1994) considered the linear stability of a suspension of aerotactic bacteria with its upper surface open to the atmosphere. The bacteria are aerobic and consume oxygen dissolved in the water. In turn oxygen diffuses into the fluid from the air through the open surface and thus a gradient of dissolved oxygen is formed. The bacteria swim up the resulting oxygen gradient in the fluid, accumulating in a dense layer at the upper surface (where oxygen is plentiful), which ultimately triggers an overturning instabilty. The basic initial equilibrium states are different in the shallow and deep layer versions of the problem (Hillesdon, Pedley \& Kessler 1995). When the layer is shallow all the bacteria in the suspension swim to the upper surface, but when the fluid layer is very deep a layer of oxygen-starved, non-motile bacteria remains at the bottom of the fluid. In the latter case the result is that a gravitationally unstable region of bacterial cells and 
fluid overlies a stable one. In this instance the linear stability analysis predicted that the onset of instability was always oscillatory.

The model we shall use is essentially the continuum model proposed by CLS which consists of equations describing the flux of cells and the fluid dynamics of the system. The major difference occurs in the expression used for cell swimming velocity which, since the cells are phototactic, is a function of the light intensity reaching the cells' light sensing organelles (photoreceptors). For uniform illumination from above in a layer of finite depth, the basic steady state is one where there is a balance between phototaxis coupled with suspension shading (resulting in up swimming, or positive phototaxis, in the lower regions of the fluid and down swimming, or negative phototaxis, in the upper regions of the fluid) and diffusion of cells in the suspension which models the random component of their swimming velocities. This results in a horizontal, concentrated layer of algae (the sublayer), the position of which depends on the light intensity. For low intensities it is close to the top of the layer, whilst for high intensities it is close to the bottom of the layer. Therefore the equilibrium cell concentration profile differs from the basic states examined by both CLS and HPK who considered exponential profiles formed at the upper surface. Only the region below the sublayer is gravitationally unstable so that when the density stratification is large enough an overturning-type instability is initiated there. The fluid motions in this region then penetrate the upper (locally) stably stratified region. This is an example of penetrative convection that occurs in a wide variety of convection problems (Straughan 1993).

The paper is organized as follows. The model for phototaxis and suspension shading is formulated in $\$ 2$. The equations and boundary conditions are outlined in $\$ 3$, and in $\$ 4$ the steady, equilibrium solution is obtained. In $\$ 5$ the equations are linearized about the basic state and asymptotic expansions are used to find the neutral curves for small-amplitude, long-wavelength perturbations to the basic state in a shallow layer. Neutral curves for the full problem are obtained numerically in $\$ 6$ and used to predict the critical cell concentrations required to trigger convective motions and the initial pattern wavelengths. The results of the model are discussed in $\$ 7$.

\section{Phototaxis and shading in suspensions of algae}

Consider a suspension of phototactic algal cells subject to illumination from vertically above. Hill \& Häder (1996) have shown from experiments that the trajectories of individual swimming cells can be described by a correlated, biased, random walk in the the limit as the time step tends to zero. From this approach, the probability density function, $f(\widehat{p})$, for the orientation vector $\widehat{p}$, a unit vector corresponding to the direction of swimming, can be shown to satisfy a Fokker-Planck equation, as was postulated by Pedley \& Kessler (1990), with coefficients determined from the data on the swimming trajectories. $f(\widehat{p})$ can be measured directly for particular parameter values but the Fokker-Planck equation provides greater insight and results for all parameter values, and allows us to calculate quantities which are difficult to measure, such as diffusion due to the random nature of the cells' motion. From $f(\hat{\boldsymbol{p}})$, we can in principle calculate the ensemble average, $\bar{p}$, of the swimming direction for all the cells in a small volume, $\delta V$, centred on a position $x^{*}$, which will be subject to approximately the same conditions. (Note that $\bar{p}$ is not a unit vector: when $|\bar{p}|=0$, there is no preferred swimming direction, and when $|\overline{\boldsymbol{p}}|=1$ all the cells in $\delta V$ swim in the same direction.) For many species of microorganisms (Hill \& Häder 1996), the swimming speed is independent of illumination, position, time and direction, and we 


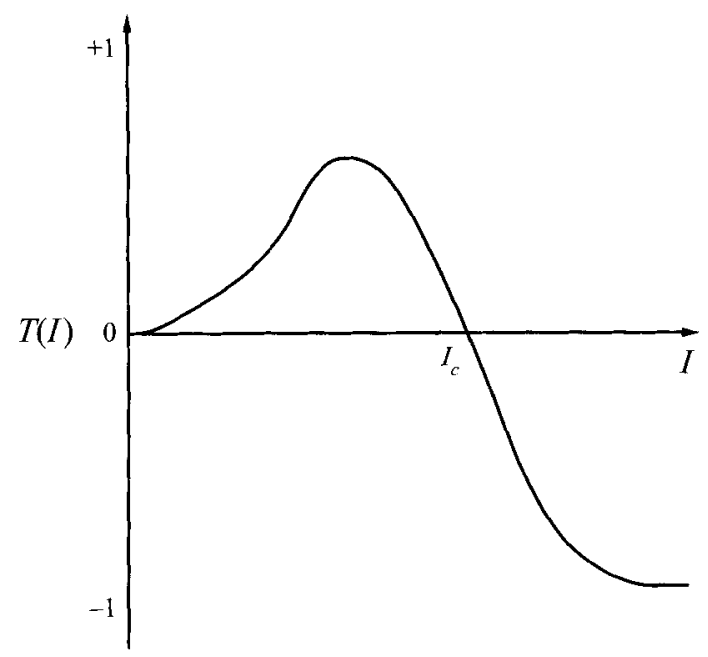

FIGURE 1. A sketch of the taxis response, $T(I)$, as a function of light intensity, $I$, for a typical phototactic algal cell. When the light is weak $T(I)$ is positive and the mean swimming direction is towards the light. At the optimum or critical intensity, $I_{c}$, there is no preferred swimming direction, and when the light intensity is very strong the mean swimming direction is away from the source.

denote the ensemble-average cell swimming speed by $V_{A}$. The average cell swimming velocity in $\delta V$ is thus

$$
\boldsymbol{V}_{\mathrm{c}}=V_{A} \overline{\boldsymbol{p}}=V_{A} \int \widehat{\boldsymbol{p}} f(\widehat{\boldsymbol{p}}) \mathrm{d} \widehat{\boldsymbol{p}},
$$

where the integral is over the surface of the unit sphere.

Many species of phototactic algae are both positively and negatively phototactic, so that at low light intensities they swim towards the light source, and vice versa. It is possible to imagine a simple experiment on a population of motile algae illuminated from vertically above. By adjusting the light intensity and measuring the proportion of the population that swim toward or away from the light, we would obtain a typical schematic intensity/response curve (figure 1). At some critical intensity, $I_{c}$ say, there is a transition between positive and negative phototaxis. Let $\widehat{\boldsymbol{k}}$ be a unit vector in the $z^{*}$-direction, vertically up. In the absence of any fluid motion, the average swimming velocity for the cells in $\delta V$ at $\boldsymbol{x}^{*}$ receiving light of intensity $I$ from vertically above can be written as

$$
\boldsymbol{V}_{c}=V_{A} \overline{\boldsymbol{p}} \quad \text { where } \quad \overline{\boldsymbol{p}}=T(I) \widehat{\boldsymbol{k}}
$$

and the taxis function $T(I)$ is a function of light intensity $I$ at $\boldsymbol{x}^{*}$ such that

$$
T(I) \begin{cases}\geqslant 0 & \text { if } I\left(x^{*}\right) \leqslant I_{c} \\ <0 & \text { if } I\left(x^{*}\right)>I_{c}\end{cases}
$$

The exact functional form of $T$ will depend on the species of algae.

For a suspension of algae subject to unidirectional illumination, the light intensity at any position is a function of cell concentration because microorganisms nearer to the light source shade those that are further away. If we assume that:

(a) the cells are homogeneously coloured particles of arbitrary shape, each possessing the same light transmittance $T_{p}$ in all orientations;

(b) the light scattering in the suspension is weak;

(c) if $\phi$ is the microorganisms' volume fraction, $\phi\left(1-T_{p}\right) \ll 1$; 
then the light intensity, $I\left(\boldsymbol{x}^{*}\right)$, received by the photoreceptors of an algal cell at position $x^{*}$ is

$$
I\left(x^{*}\right)=I_{s} \exp \left(-\alpha^{*} \int_{0}^{r^{*}} n^{*}\left(r^{\prime}\right) \mathrm{d} r^{\prime}\right) .
$$

Here $\boldsymbol{r}^{*}$ is the vector from the cell to the light source of intensity $I_{s}$ and $n^{*}\left(\boldsymbol{x}^{*}\right)$ is the concentration of cells at any position $\boldsymbol{x}^{*}$. $\alpha^{*}$ is the extinction coefficient, which quantifies the strength of absorption and scattering of light in the suspension. This expression is derived from the Lambert-Beer law for weak scattering in a solution (Herdan 1960). Kessler (1989) was the first to suggest that the Lambert-Beer law could be used as the basis of a model for algal phototaxis, although it has been used previously to calculate absorption in algal suspensions (Duysens 1956). This then is the expression we will use to describe absorption in our model. All the effects of multiple scattering have been neglected, so an algal cell only perceives the light that is travelling to it in a direct line from the source.

It is also important to consider whether (2.4) would give the actual intensity perceived by an algal cell at $\boldsymbol{x}^{*}$ at a particular instant. Most algae rotate with frequencies of $1-2 \mathrm{~Hz}$ as they swim, and so it is possible to visualize their photoreceptors as antennae that scan the light intensity field as the cell's body revolves (Foster \& Smyth 1980). Thus $I\left(\boldsymbol{x}^{*}\right)$ is the light intensity perceived by the cell over several rotations during its forward motion and the timescale for changes in the light intensity must be longer than the time required for the cell to detect those changes. Such an assumption is reasonable for bioconvection patterns that evolve and develop on timescales of tens of seconds.

\section{The continuum model}

In common with all the previous models of bioconvection we shall assume that the suspension of algae can be treated as a continuous distribution. We also assume that the lengthscale of the bulk motions and the concentration distribution are large compared to typical cell diameters and cell spacing. The algal cells themselves are modelled as internally homogeneous, pigmented particles of volume $v$ and density $\rho+\Delta \rho(\Delta \rho \ll \rho$, where $\rho$ is the density of the fluid) and possess the same light transmittance in all orientations. The number of cells in a small volume $\delta \mathrm{V}$ defined relative to the Cartesian axis $O x^{*} y^{*} z^{*}$ is $n^{*}\left(x^{*}, t^{*}\right) \delta V$, where $z^{*}$ is the axis in the vertical direction and $t^{*}$ is time. Neglecting all inertia in the cells' motion and supposing that the suspension is dilute $\left(n^{*} v \ll 1\right)$ and incompressible then, if $\boldsymbol{u}^{*}\left(\boldsymbol{x}^{*}, t^{*}\right)$ is the average velocity of all the material in $\delta \mathrm{V}$,

$$
\nabla^{*} \cdot \boldsymbol{u}^{*}=0
$$

For simplicity, we shall assume that the effect of the cells on the suspension is dominated by the stokeslets due to their negative buoyancy and that all other contributions to the bulk stress are sufficiently small to be neglected. For estimates of the various additional contributions, see Pedley \& Kessler (1990). Therefore, for such a suspension of cells and neglecting all forces on the fluid except the cells' negative buoyancy, $n^{*} v g \Delta \rho \delta V$ where $g$ is the acceleration due to gravity, the momentum equation under the Boussinesq approximation is

$$
\rho \frac{\mathrm{D} \boldsymbol{u}^{*}}{\mathrm{D} t^{*}}=-\nabla p_{e}-n^{*} v g \Delta \rho \widehat{\boldsymbol{k}}+\mu \nabla^{* 2} \boldsymbol{u}^{*}
$$




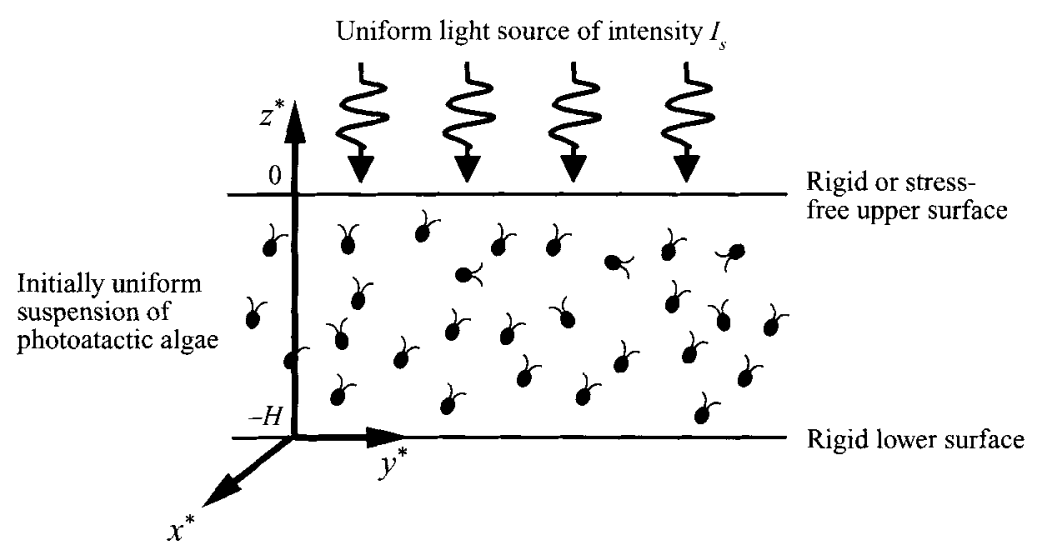

FIGURE 2. The geometry of the problem under consideration.

$\mathrm{D} / \mathrm{D} t^{*}=\partial / \partial t^{*}+\boldsymbol{u}^{*} \cdot \nabla^{*}$ is the material time derivative, $\widehat{\boldsymbol{k}}$ is a unit vector in the $z^{*}$-direction, $p_{e}$ is the excess pressure above hydrostatic and $\mu$ is the dynamic viscosity of the suspension which, since the suspension is dilute, is considered to be that of the fluid, which is effectively water.

The equation for cell conservation is

$$
\frac{\partial n^{*}}{\partial t^{*}}=-\nabla^{*} \cdot J^{*}
$$

where $\boldsymbol{J}^{*}\left(\boldsymbol{x}^{*}, t^{*}\right) \delta \boldsymbol{S}$ is the net flux across a surface element, $\delta \boldsymbol{S}$, at $\boldsymbol{x}^{*} . \boldsymbol{J}^{*}$ can be written as

$$
\boldsymbol{J}^{*}=n^{*} \boldsymbol{u}^{*}+n^{*} V_{c} \overline{\boldsymbol{p}}-\boldsymbol{D}^{*} \cdot \nabla^{*} n^{*}
$$

where $\bar{p}$ is given by (2.1). The first term, $n^{*} \boldsymbol{u}^{*}$, is the flux due to advection of cells by the bulk flow. The other terms represent fluxes arising from the stochastic nature of the cells' swimming: $n^{*} V_{c} \overline{\boldsymbol{p}}$ is a mean flux due to cell swimming and $-\boldsymbol{D}^{*} \cdot \nabla^{*} n^{*}$ is a diffusive flux of cells down cell concentration gradients.

We shall consider a fluid layer with horizontal boundaries at $z^{*}=-H, 0$ and shall assume that the vertical boundaries are far enough away that the layer has an effectively infinite width (see figure 2). The suspension is illuminated by parallel light from a uniform source of intensity $I_{s}$ vertically above the layer. Two key ad hoc assumptions have been made to simplify the expression (3.4) for the cell flux. Firstly, the cells are assumed to be purely phototactic in that $\bar{p}$ is always parallel to $\widehat{\boldsymbol{k}}$ and the effects of any net viscous torques due to local fluid velocity gradients, which might induce a non-zero horizontal component of the mean swimming velocity, have been neglected. Clearly, this is a significant assumption but we justify it on the grounds that phototaxis is in general a strong active orientation mechanism, so that swimming cells correct very rapidly for external torques. Thus 'pure phototaxis' is a valid limiting case to consider in order to understand the complexities of the effects of phototaxis on bioconvection before moving on to more detailed models. Secondly, we have assumed that the diffusion tensor, $\boldsymbol{D}$, which should be derived from a swimming velocity autocorrelation function, is a constant orthotropic tensor of the form

$$
\mathbf{D}=\left(\begin{array}{ccc}
D_{H} & 0 & 0 \\
0 & D_{H} & 0 \\
0 & 0 & D_{V}
\end{array}\right)
$$


This is likely to make a quantitative rather than qualitative difference compared with a more rational model, as some diffusion is always present due to the randomness in the cells' swimming. Since we have no method of estimating the ratio of $D_{H}$ to $D_{V}$ (the horizontal to vertical diffusion parameters) at present, we shall arbitrarily take $D_{H}=D_{V}$ for most of the calculations in this paper, although $D_{H}$ and $D_{V}$ are retained in the analysis for future use. The two simplifications allow us to eliminate the Fokker-Planck equation from the governing equations.

If light absorption by the pure fluid is negligible (which is a reasonable assumption since we are interested in suspensions at most a few centimetres deep) then, as we showed in $\$ 2$, the light intensity received by a cell at $\boldsymbol{x}^{*}=\left(x^{*}, y^{*}, z^{*}\right)$ is, from (2.4),

$$
I\left(\boldsymbol{x}^{*}\right)=I_{s} \exp \left(-\alpha^{*} \int_{z^{*}}^{0} n^{*}\left(\boldsymbol{x}^{\prime}, t^{*}\right) \mathrm{d} z^{\prime}\right) .
$$

Provided that the absorption across the suspension is weak $\left(0<\alpha^{*} n^{*} H \ll 1\right)$ and that the illumination across the layer $I_{s}$ is close to the critical intensity $I_{c}$, which represents the transition from positive to negative phototaxis, then $T(I)$ (see (2.3)) is approximately linear so that from $(2.2)$

$$
\overline{\boldsymbol{p}}=-A\left(I-I_{c}\right) \widehat{\boldsymbol{k}} .
$$

If the critical intensity $I_{c}$ occurs at position $z^{*}=-C H(0 \leqslant C \leqslant 1)$ for a vertically uniform concentration profile $N_{0}$ then, for cells at $x^{*}$, correct to $O\left(\alpha^{*} n^{*} H\right)$

$$
\overline{\boldsymbol{p}}=A \boldsymbol{I}_{c} \alpha^{*}\left(\int_{z^{*}}^{0} n^{*}\left(\boldsymbol{x}^{\prime}, t^{*}\right) \mathrm{d} z^{\prime}-C H N_{0}\right) \widehat{\boldsymbol{k}} .
$$

The continuum model is now complete.

\section{The steady solution}

In our analysis we shall present results for both free and rigid upper horizontal boundaries. Indeed, in experiments it is usually the case that the lower boundary is rigid, whilst the upper boundary may be free or rigid. HPK considered only rigid upper and lower boundaries since experiments indicated that the introduction of a partial rigid surface on top of the fluid layer did not affect the bioconvection patterns. It is unlikely that any free surface is ever fully stress-free (there is always surface tension for example), but our results indicate that the behaviour of a suspension with a stress free upper surface should differ significantly from one with a rigid upper boundary, initially at least.

The boundary conditions for the problem are

$$
\begin{aligned}
\boldsymbol{u}^{*} \cdot \widehat{\boldsymbol{k}}=0 & \text { on } & z^{*}=-H, 0, \\
\boldsymbol{J}^{*} \cdot \widehat{\boldsymbol{k}}=0 & \text { on } & z^{*}=-H, 0 .
\end{aligned}
$$

For a rigid boundary

$$
\boldsymbol{u}^{*} \times \widehat{\boldsymbol{k}}=0 \quad \text { on } \quad z^{*}=-H, 0,
$$

whilst for a free boundary

$$
\frac{\partial^{2}}{\partial z^{* 2}}\left(\boldsymbol{u}^{*} \cdot \widehat{\boldsymbol{k}}\right)=0 \quad \text { on } \quad z^{*}=-H, 0
$$


When there is no fluid motion the steady equation for cell flux becomes (on substituting (3.4) and (3.8) into (3.3)),

$$
\frac{\mathrm{d}}{\mathrm{dz}}\left\{n^{*} V_{A} A I_{c} \alpha^{*}\left(\int_{z^{*}}^{0} n\left(x^{\prime}\right) \mathrm{d} z^{\prime}-C H N_{0}\right)-D_{V} \frac{\mathrm{d} n^{*}}{\mathrm{~d} z^{*}}\right\}=0 .
$$

Integrating with respect to $z^{*}$ and applying the boundary condition (4.2), we obtain

$$
\frac{\mathrm{d} n^{*}}{\mathrm{~d} z^{*}}-\frac{V_{A} A I_{c} \alpha^{*} N_{0} H}{D_{V}} n^{*}\left(\frac{1}{N_{0} H} \int_{z^{*}}^{0} n^{*}\left(\boldsymbol{x}^{\prime}\right) \mathrm{d} z^{\prime}-C\right)=0 .
$$

The solution of this equation is (see the Appendix)

$$
n^{*}\left(z^{*}\right)=\frac{K^{* 2} D_{V}}{2 V_{A} \bar{A} I_{c} \alpha^{*}} \operatorname{sech}^{2}\left(\frac{1}{2} K^{*}\left(z^{*}+C H\right)\right),
$$

where $K^{*}$ is a constant of integration which can be related to $N_{0}$, the number of cells per unit volume for the whole layer, by the relation

$$
\int_{-H}^{0} n^{*}\left(x^{\prime}\right) \mathrm{d} z^{\prime}=N_{0} H
$$

The basic state described by (4.7) is not the same as the one analysed previously by CLS or HPK since the cell accumulation can be anywhere within the layer, not just at the top of the suspension. When the cell aggregation is not at the top of the layer, it is apparent from (4.7) that the region above $z^{*}=-C H$ is locally gravitationally stable and convective motions occurring in the unstable region below $z^{*}=-C H$ will penetrate the upper layer. Penetrative convection arises in a wide variety of physical phenomena from the convective motions in stars to ocean dynamics, but it was introduced in a paper by Veronis (1963) on the stability of a layer of water, the bottom of which is maintained at $0^{\circ} \mathrm{C}$ and the top at a temperature greater than $4^{\circ} \mathrm{C}$. This results in a density stratification with a gravitationally stable layer overlying an unstable layer which, in this aspect at least, is analogous to the problem treated in this paper. A thorough review of all the mathematical aspects of penetrative convection along with an extensive bibliography may be found in Straughan (1993).

The final step before linearizing our system of equations about the equilibrium state is to non-dimensionalize it. All lengths are scaled on $H$ the depth of the layer, the bulk fluid velocity on $D_{V} / H$, time on the diffusive timescale $H^{2} / D_{V}$, cell concentration on $N_{0}$ the uniform cell concentration, diffusion on the vertical diffusion parameter $D_{V}$, and the pressure $p_{e}$ on $v D_{V} \rho / H^{2}$, where $v$ is the kinematic viscosity. Non-dimensional quantities will be represented by unstarred symbols. In terms of the new symbols the basic equilibrium state becomes

$$
\boldsymbol{u}=0, \quad n(z)=\frac{K^{2}}{2 d} \operatorname{sech}^{2}\left(\frac{1}{2} K(z+C)\right),
$$

where the horizontal boundaries are at $z=-1,0$ and

$$
d=\frac{H V_{A} P}{D_{V}} \quad \text { where } \quad P=A I_{c} \alpha^{*} N_{0} H .
$$

$K=K^{*} H$ and is determined by (4.8) which produces the transcendental equation

$$
K\left[\tanh \left(\frac{1}{2} K C\right)-\tanh \left(\frac{1}{2} K(C-1)\right)\right]=d .
$$

Since $P$ is directly proportional to $\alpha^{*}, I_{c}$ and $A$, it represents the strength of the 
phototaxis in the suspension (see (3.8)); $d$ is the ratio of the layer depth $H$ to $l=D_{V} / V_{A} P$, the depth scale for the cell (hence density) accumulation which forms at $z=-C$. This is not immediately clear from (4.9), but if we consider the special case $K \ll 1$ then (4.11) can be solved approximately to give $K \simeq(2 d)^{1 / 2}(d \ll 1)$ so that (4.9) becomes

$$
n(z)=\operatorname{sech}^{2}\left(\left(\frac{1}{2} d\right)^{1 / 2}(z+C)\right) .
$$

Whether the basic equilibrium state detailed above is ever achieved in reality is an important question which we shall defer to the discussion. Until then we shall assume that the suspension is initially well mixed so that the cell concentration is uniform and that any residual fluid motions are quickly damped out. In this case the cells will accumulate in a horizontal layer centred at $z=-C$, where the light intensity measured by their photoreceptors is equal to $I_{c}$, and form the steady equilibrium state given by (4.9). We now analyse the stability of this basic state to determine under what circumstances bioconvection will begin, the initial size of the resulting patterns and the underlying physical mechanisms. The stability will be shown to depend on four dimensionless parameters: the horizontal wavenumber $k$ of the disturbance, $d$ which is the ratio of the depth of the layer to that of the sublayer, the position of the sublayer $C$, and a Rayleigh number defined in the next section, which is proportional to the uniform cell concentration $N_{0}$.

\section{The linear stability problem}

We shall consider a small perturbation to the equilibrium state (4.9) of amplitude $\epsilon$ where $0<\epsilon \ll 1$. In this case

$$
\boldsymbol{u}=\epsilon \boldsymbol{u}_{1}, \quad n=n_{0}+\epsilon n_{1}(\boldsymbol{x}, t) \quad \text { where } \quad n_{0}=\frac{K^{2}}{2 d} \operatorname{sech}^{2}\left(\frac{1}{2} K(z+C)\right) .
$$

If $\boldsymbol{u}_{1}=\left(u_{1}, v_{1}, w_{1}\right)$ and we define $D_{H}=\kappa D_{V}$ where $\kappa$ is a positive real number then, substituting the above perturbation quantities into (3.1), (3.2) and (3.3) and linearizing about the basic state by collecting the $O(\epsilon)$ terms, gives

$$
\begin{gathered}
\nabla \cdot \boldsymbol{u}_{1}=0 \\
S_{c}^{-1} \frac{\partial \boldsymbol{u}_{1}}{\partial t}=-\nabla p_{e}-\beta n_{1} \widehat{\boldsymbol{k}}+\nabla^{2} \boldsymbol{u}_{1} \\
\frac{\partial n_{1}}{\partial t}+d\left(\frac{\mathrm{d} n_{0}}{\mathrm{~d} z} \int_{z}^{0} n_{1}(x, y, s, t) \mathrm{d} s+\left(\int_{z}^{0} n_{0}(s) \mathrm{d} s-C\right) \frac{\partial n_{1}}{\partial z}-2 n_{0} n_{1}\right) \\
-\kappa \nabla_{h}^{2} n_{1}-\frac{\partial^{2} n_{1}}{\partial z^{2}}=-w_{1} \frac{\mathrm{d} n_{0}}{\mathrm{~d} z},
\end{gathered}
$$

where

$$
\beta=\frac{N_{0} v \Delta \rho g H^{3}}{\rho D_{V} v} .
$$

$S_{c}=v / D_{V}$ is the Schmidt number and $\nabla_{h}^{2}=\partial^{2} / \partial x^{2}+\partial^{2} / \partial y^{2} ; p_{e}$ and the horizontal component of $\boldsymbol{u}$ may be eliminated from the above equations by taking the curl of (5.3) twice and retaining the $z$-component of the result. This reduces the system to two equations in $w_{1}$ and $n_{1}$. These quantities can then be resolved into normal modes 
by the substitutions

$$
n_{1}=\Phi(z) f(x, y) \exp (\sigma t), \quad w_{1}=W(z) f(x, y) \exp (\sigma t),
$$

where the horizontal planform $f$ satisfies $\nabla_{h}^{2} k=-k^{2} f, k$ being a dimensionless horizontal wavenumber, and $\sigma$ is the growth rate. Using (5.6) the governing equations become

$$
\begin{gathered}
\left(\sigma S_{c}^{-1}+k^{2}-\frac{\mathrm{d}^{2}}{\mathrm{~d} z^{2}}\right)\left(k^{2}-\frac{\mathrm{d}^{2}}{\mathrm{~d} z^{2}}\right) W=-\beta k^{2} \Phi \\
d \frac{\mathrm{d} n_{0}}{\mathrm{~d} z} \int_{z}^{0} \Phi(s) \mathrm{d} s+\left\{\left(\sigma-2 d n_{0}+\kappa k^{2}\right)+d\left(\int_{z}^{0} n_{0}(s) \mathrm{d} s-C\right) \frac{\mathrm{d}}{\mathrm{d} z}-\frac{\mathrm{d}^{2}}{\mathrm{~d} z^{2}}\right\} \Phi=-\frac{\mathrm{d} n_{0}}{\mathrm{~d} z} W
\end{gathered}
$$

subject to the boundary conditions

$$
\frac{\mathrm{d} \Phi}{\mathrm{d} z}-d\left\{\left(\int_{z}^{0} n_{0}(s) \mathrm{d} s-C\right) \Phi+n_{0} \int_{z}^{0} \Phi(s) \mathrm{d} s\right\}=0 \quad \text { at } \quad z=-1,0
$$

and for rigid boundaries

$$
W=\frac{\mathrm{d} W}{\mathrm{~d} z}=0 \quad \text { at } \quad z=-1,0 .
$$

At a stress-free surface the last condition is replaced by

$$
W=\frac{\mathrm{d}^{2} W}{\mathrm{~d} z^{2}}=0 .
$$

Similar equations to (5.7) and (5.8) were derived by CLS and HPK for the linear stability problems involving up swimming and gyrotaxis. The momentum equation (5.7) and the right-hand side of the cell flux equation (5.8) are identical to those used previously. The new aspects of this model, the effects of phototactic motions, are incorporated in the $\left(\mathrm{d} n_{0} / \mathrm{d} z\right) \int_{z}^{0} \Phi \mathrm{d} s, n_{0} \Phi$ and $\left(\int_{z}^{0} n_{0}(s) \mathrm{d} s-C\right) \mathrm{d} \Phi / \mathrm{d} z$ terms found on the left-hand side of the cell flux equation. Equation (5.8) is a linear integrodifferential equation with non-constant coefficients, the solution of which represents a problem of considerable difficulty. Fortunately it may be reduced to a linear ODE (at the cost of increasing its order by one) by use of the substitution

$$
\Phi^{*}(z)=\int_{z}^{0} \Phi(s) \mathrm{d} s
$$

in which case $\Phi^{* \prime}(z)=-\Phi(z)$ etc. Equations (5.7) and (5.8) become

$$
\begin{gathered}
\left(\sigma S_{c}^{-1}+k^{2}-\frac{\mathrm{d}^{2}}{\mathrm{~d} z^{2}}\right)\left(k^{2}-\frac{\mathrm{d}^{2}}{\mathrm{~d} z^{2}}\right) W=\beta k^{2} \frac{\mathrm{d} \Phi^{*}}{\mathrm{~d} z} \\
{\left[d \frac{\mathrm{d} n_{0}}{\mathrm{~d} z}-\left(\sigma-2 d n_{0}+\kappa k^{2}\right) \frac{\mathrm{d}}{\mathrm{d} z}-d\left(\int_{z}^{0} n_{0}(s) \mathrm{d} s-C\right) \frac{\mathrm{d}^{2}}{\mathrm{~d} z^{2}}+\frac{\mathrm{d}^{3}}{\mathrm{~d} z^{3}}\right] \Phi^{*}=-\frac{\mathrm{d} n_{0}}{\mathrm{~d} z} W .}
\end{gathered}
$$

The boundary conditions remain the same except (5.9) which becomes

$$
\frac{\mathrm{d}^{2} \Phi^{*}}{\mathrm{~d} z^{2}}-d\left\{\left(\int_{z}^{0} n_{0}(s) \mathrm{d} s-C\right) \frac{\mathrm{d} \Phi^{*}}{\mathrm{~d} z}-n_{0} \Phi^{*}\right\}=0 \quad \text { at } \quad z=-1,0 .
$$

The system of equations (5.13)-(5.15) is seventh order, as opposed to the original 
system which was sixth order, and therefore an additional boundary condition is now required. This is easily supplied by observing from (5.12) that

$$
\Phi^{*}(z)=0 \quad \text { at } \quad z=0 .
$$

The normal-modes equations may now be solved in precisely the same way as those which arise in other convective instability problems such as Rayleigh-Bénard convection. We shall define the Rayleigh number for the problem as

$$
R=\beta d=\frac{N_{0} v \Delta \rho g H^{3} d}{\rho D_{V} v}
$$

which is appropriate because it represents the ratio of the buoyancy forces which drive the convective instability (since the cell accumulation in the sublayer is proportional to $\left.N_{0} d\right)$ to the viscous forces that inhibit it. A more usual definition for Rayleigh number would depend on the concentration difference across the layer, or in this case the maximum cell concentration in the sublayer $\left(N_{s}\right)$. To be consistent with previous authors on bioconvection, we should use such a Rayleigh number, but unfortunately this proves to be problematic since the solution to the transcendental equation (4.11) is required to calculate $N_{s}$.

The aim of our analysis is to determine for what values of $d, k, C$ and $R$ the basic state becomes unstable for both rigid and stress-free upper boundaries (the lower boundary is assumed to be rigid) by plotting neutral curves in the $(k, R)$ plane. A neutral curve is defined as the locus of points where $\operatorname{Re}(\sigma)=0$. If in addition $\operatorname{Im}(\sigma)=0$ on such a curve then the principle of exchange of stabilities is said to be valid (Chandrasekhar 1961) and the perturbation is stationary or non-oscillatory. Alternatively, if $\operatorname{Im}(\sigma) \neq 0$ then oscillatory or overstable solutions might exist. Oscillatory instabilities arise in many situations, often when there is competition between a stabilizing and a destabilizing process. CLS were able to show that the convective instability caused by pure up swimming was always stationary, although a two-dimensional numerical model of up-swimming motile particles indicated the possibility of oscillatory solutions (Childress \& Peyret 1976). HPK found evidence of oscillatory instabilities driven by the gyrotactic response of the microorganisms to the shear at the upper boundary of the layer, although they proved that the instability was stationary for two stress-free boundaries. We have been unable to prove exchange of stability for this problem, which is explained by the surprising result that the numerical analysis has revealed the existence of oscillatory solutions for certain ranges of $d$ and $C$ for both rigid and stress-free upper surfaces. Results and a physical explanation for the occurrence of overstability are presented in $\$ 6.2$.

For any given values of $C$ and $d$, there is an infinite number of branches of the neutral curve $R^{(n)}(k)(n=1,2,3 \ldots)$ each one corresponding to a different solution of the linear stability problem. The solution branch of most interest is the one on which $R$ has its minimum value $\left(k_{c}, R_{c}\right)$. This is the most unstable solution from which the wavelength of the initial disturbance may be obtained using the relation $\lambda_{c}=2 \pi / k_{c}$. It is important to remember that we expect the linear stability theory to describe only the initial pattern spacing before the nonlinear effects in the system become significant. Solutions consist of layers of convection cells stacked vertically one on another. A solution is said to be mode $n$ if it has $n$ layers (or convection cells). Often, although not always, the most unstable solution corresponds to the $R^{(1)}(k)$ branch of the neutral curve and is mode 1 . 


\subsection{An asymptotic solution}

In this subsection we shall present an asymptotic solution to the normal-modes problem (5.13)-(5.15) and (5.10)-(5.11) for rigid and stress-free upper surfaces when $0<d \ll 1$. Physically this corresponds to a shallow layer approximation when the scale for the equilibrium cell distribution $l$ is large compared to the depth of the layer $H$. The reason for presenting this analysis is that it provides an insight into the fluid mechanics of bioconvection and a useful check of the numerical solutions. Both CLS and HPK were also able to consider the deep layer limit $(d \gg 1)$ in their asymptotic analyses, in which a concentrated aggregation of cells forms at the top of the fluid in a boundary layer and the stability can be analysed using the method of matched asymptotic expansions. It is possible to consider a similar case for this problem, but unfortunately the resulting equations do not appear to be tractable analytically.

We have already mentioned, in the case when $d \ll 1$, that (4.11) can be solved approximately to give $K \simeq(2 d)^{1 / 2}$ so that $n(z)$ is given by (4.9). Motivated by the studies of CLS and HPK, we anticipate that for this shallow layer case the most unstable wavelength is zero when $C=0$. Thus we consider small wavenumbers such that $k \sim d$ and we can rewrite (5.13) and (5.14) as

$$
\left(\sigma S_{c}^{-1}+d^{2} \tilde{k}^{2}-\frac{\mathrm{d}^{2}}{\mathrm{~d} z^{2}}\right)\left(d^{2} \tilde{k}^{2}-\frac{\mathrm{d}^{2}}{\mathrm{~d} z^{2}}\right) W=d R \tilde{k}^{2} \frac{\mathrm{d} \Phi^{*}}{\mathrm{~d} z}
$$

and

$$
\left[d \frac{\mathrm{d} n_{0}}{\mathrm{~d} z}-\left(\sigma-2 d n_{0}+\kappa d^{2} \tilde{k}^{2}\right) \frac{\mathrm{d}}{\mathrm{d} z}-d\left(\int_{z}^{0} n_{0}(s) \mathrm{d} s-C\right) \frac{\mathrm{d}^{2}}{\mathrm{~d} z^{2}}+\frac{\mathrm{d}^{3}}{\mathrm{~d} z^{3}}\right] \Phi^{*}=-\frac{\mathrm{d} n_{0}}{\mathrm{~d} z} W,
$$

where $\tilde{k}=k / d$ so $\tilde{k} \sim 1$. On expanding the hyperbolic functions as power series in $d$, we obtain

$$
\begin{aligned}
n_{0} & =1+d s_{1}(z)+d^{2} s_{2}(z)+O\left(d^{3}\right), \\
\frac{\mathrm{d} n_{0}}{\mathrm{~d} z} & =d \frac{\mathrm{d} s_{1}}{\mathrm{~d} z}+d^{2} \frac{\mathrm{d}^{2} s_{2}}{\mathrm{~d} z^{2}}+O\left(d^{3}\right), \\
\int_{z}^{0} n_{0}\left(z^{\prime}\right) \mathrm{d} z^{\prime}-C & =-(z+C)+d \int_{z}^{0} s_{1}\left(z^{\prime}\right) \mathrm{d} z^{\prime}+d^{2} \int_{z}^{0} s_{2}\left(z^{\prime}\right) \mathrm{d} z^{\prime}+O\left(d^{3}\right),
\end{aligned}
$$

where

$$
\begin{aligned}
& s_{1}(z)=-\frac{1}{2}\left(z^{2}+2 z C+C-\frac{1}{3}\right), \\
& s_{2}(z)=\frac{1}{6}\left(z^{4}+4 z^{3} C+3 z^{2} C^{2}-2 z C^{3}+3 z^{2} C+6 z C^{2}-C^{3}-z^{2}-2 z C+2 C^{2}-C+\frac{2}{15}\right) .
\end{aligned}
$$

There are of course many leading-order balances which in principle at least are solvable by elementary methods, but in general it turns out that they either reduce to the balance considered below or ultimately require a numerical solution. HPK noted that to obtain non-trivial solutions, which satisfy the seven leading-order boundary conditions, requires that the highest derivatives in (5.18) and (5.19) are retained and the leading-order balance in (5.18) must give

$$
\left(\frac{\mathrm{d}^{2}}{\mathrm{~d} z^{2}}-\sigma S_{c}^{-1}\right) \frac{\mathrm{d}^{2} W}{\mathrm{~d} z^{2}}=d \tilde{k}^{2} R \frac{\mathrm{d} \Phi^{*}}{\mathrm{~d} z}
$$

as we might expect, since (5.20) represents the balance between the viscous terms on the left-hand side and the buoyancy terms on the right-hand side which drive the 
flow. Without loss of generality we also specify that

$$
\Phi^{*}=O(1)
$$

Rigid upper surface

To be consistent with (5.20) and (5.21) we shall examine the case

$$
\Phi^{*} \sim 1, \quad W \sim d, \quad R \sim 1, \quad \sigma \sim 1,
$$

and expand these quantities as power series in $d$ :

$$
\Phi^{*}=\sum_{n=0}^{\infty} d^{n} \Phi_{n}^{*}, \quad W=\sum_{n=1}^{\infty} d^{n} W_{n}, \quad R=\sum_{n=0}^{\infty} d^{n} R_{n}, \quad \sigma=\sum_{n=0}^{\infty} d^{n} \sigma_{n} .
$$

This produces the leading-order problem

$$
\begin{gathered}
\frac{\mathrm{d}^{4} W_{1}}{\mathrm{~d} z^{4}}-\sigma_{0} S_{c}^{-1} \frac{\mathrm{d}^{2} W_{1}}{\mathrm{~d} z^{2}}=\tilde{k}^{2} R_{0} \frac{\mathrm{d} \Phi_{0}^{*}}{\mathrm{~d} z}, \\
\frac{\mathrm{d}^{3} \Phi_{0}^{*}}{\mathrm{~d} z^{3}}-\sigma_{0} \frac{\mathrm{d} \Phi_{0}^{*}}{\mathrm{~d} z}=0,
\end{gathered}
$$

subject to the boundary conditions (for a rigid upper surface)

$$
\frac{\mathrm{d}^{2} \Phi_{0}^{*}}{\mathrm{~d} z^{2}}=W_{1}=\frac{\mathrm{d} W_{1}}{\mathrm{~d} z}=0 \quad \text { at } \quad z=-1,0
$$

and

$$
\Phi_{0}^{*}=0 \quad \text { at } \quad z=0 .
$$

Equation (5.23) represents a purely diffusive balance so there are no effects from the cells' phototaxis at this order. Integrating (5.23) with respect to $z$ and applying the boundary conditions gives

$$
\frac{\mathrm{d}^{2} \Phi_{0}^{*}}{\mathrm{~d} z^{2}}-\sigma_{0} \Phi_{0}^{*}=0
$$

and $\sigma_{0}=0$ or $\Phi_{0}^{*}(-1)=0$. If $\Phi_{0}^{*}(-1)=0$ then $\Phi_{0}^{*}(z)$ has only a trivial solution if $\sigma_{0}>0$, but if $\sigma_{0}<0$ then

$$
\Phi_{0}^{*}(z)=\sin n \pi z \quad \text { where } \quad n \in \mathbb{Z}^{+} .
$$

Therefore the problem possesses an infinite number of stable modes but if $\sigma_{0}=0$ then

$$
\Phi_{0}^{*}=-z, \quad W_{1}=-\frac{1}{24} \tilde{k}^{2} R_{0}\left(z^{4}+2 z^{3}+z^{2}\right),
$$

which is precisely the same leading-order solution as that obtained by HPK where $\Phi_{0}^{*}$ has been scaled so that $\Phi_{0}=1$ without loss of generality.

At second order we have

$$
\begin{gathered}
\frac{\mathrm{d}^{4} W_{2}}{\mathrm{~d} z^{4}}-\tilde{k}^{2} R_{0} \frac{\mathrm{d} \Phi_{1}^{*}}{\mathrm{~d} z}=\sigma_{1} S_{c}^{-1} \frac{\mathrm{d}^{2} W_{1}}{\mathrm{~d} z^{2}}-\tilde{k}^{2} R_{1} \\
\frac{\mathrm{d}^{3} \Phi_{1}^{*}}{\mathrm{~d} z^{3}}-2+\sigma_{1}=0,
\end{gathered}
$$

subject to the boundary conditions

$$
\frac{\mathrm{d}^{2} \Phi_{1}^{*}}{\mathrm{~d} z^{2}}-2 z-C=W_{2}=\frac{\mathrm{d} W_{2}}{\mathrm{~d} z}=0 \quad \text { at } \quad z=-1,0
$$


which has the solution

$$
\Phi_{1}^{*}=0 \quad \text { at } \quad z=0,
$$

$$
\begin{gathered}
\Phi_{1}^{*}=\frac{1}{3} z^{3}+\frac{1}{2} C z^{2}, \\
W_{2}=\frac{1}{120} \tilde{k}^{2} R_{0}\left(\frac{1}{3} z^{6}+C z^{5}\right)-\frac{1}{24} \tilde{k}^{2} R_{1} z^{4}-\frac{1}{12} \tilde{k}^{2}\left(R_{1}+\frac{1}{15}\left(\frac{9}{2} C-2\right)\right) z^{3} \\
-\frac{1}{24} \tilde{k}^{2}\left(R_{1}+\frac{2}{5} R_{0}\left(C-\frac{1}{2}\right)\right) z^{2},
\end{gathered}
$$

provided that $\sigma_{1}=0$. The second- and higher-order systems of equations are inhomogeneous and therefore must satisfy solvability conditions. These are obtained by multiplying each system by the adjoint of the homogeneous system and requiring that the integral from $z=-1$ to 0 of the result must vanish. This is equivalent to setting the integral of the cell conservation equation (5.19) over the layer depth to zero. The solvability condition at $O\left(d^{2}\right)$ is

$$
\begin{aligned}
\int_{-1}^{0} \frac{\mathrm{d}^{3} \Phi_{2}^{*}}{\mathrm{~d} z^{3}} \mathrm{~d} z & +\int_{-1}^{0}(z+C) \frac{\mathrm{d}^{2} \Phi_{1}^{*}}{\mathrm{~d} z^{2}} \mathrm{~d} z+2 \int_{-1}^{0} \frac{\mathrm{d} \Phi_{1}^{*}}{\mathrm{~d} z} \mathrm{~d} z-\int_{-1}^{0}(z+C)^{2} \frac{\mathrm{d} \Phi_{0}^{*}}{\mathrm{~d} z} \mathrm{~d} z \\
& -\left(\sigma_{2}+\kappa \tilde{k}^{2}\right) \int_{-1}^{0} \frac{\mathrm{d} \Phi_{0}^{*}}{\mathrm{~d} z} \mathrm{~d} z-\int_{-1}^{0}(z+C) \Phi_{0}^{*} \mathrm{~d} z=\int_{-1}^{0}(z+C) W_{1} \mathrm{~d} z
\end{aligned}
$$

At $O\left(d^{3}\right)$ there is a similar but more complicated condition. Examination of (5.35) indicates that we only require the third-order boundary condition for $\Phi_{2}^{*}$ to calculate $\sigma_{2}$ which is

$$
\frac{\mathrm{d}^{2} \Phi_{2}^{*}}{\mathrm{~d} z^{2}}=0 \quad \text { at } \quad z=0, \quad \frac{\mathrm{d}^{2} \Phi_{2}^{*}}{\mathrm{~d} z^{2}}=2\left(C^{2}-2 C+1\right) \quad \text { at } \quad z=-1 .
$$

Therefore imposing the solvability condition (5.35) gives

$$
\sigma_{2}=\tilde{k}^{2}\left(\frac{R_{0}(1-2 C)}{1440}-\kappa\right) .
$$

On the neutral curve $\sigma_{2}=0$, in which case (5.37) gives

$$
R_{0}=\frac{1440 \kappa}{1-2 C} \text {. }
$$

Proceeding to the third-order problem, we find after some algebra that the $O\left(d^{3}\right)$ solvability condition yields

$$
\sigma_{3}=\frac{1}{302400}\left\{210(1-2 C) R_{1}+\left(280 C^{3}-420 C^{2}+223 C-43\right) R_{0}\right\} \tilde{k}^{2} .
$$

On the neutral curve $\sigma_{3}=0$, so that along with (5.38) we have

$$
R_{c}=\frac{1440 \kappa}{1-2 C}\left\{1+\frac{1}{210} d \frac{\left(280 C^{3}-420 C^{2}+223 C-43\right)}{2 C-1}\right\}+O\left(d^{2}\right) .
$$

Hence with this scaling there is just one neutrally stable solution that (5.28) shows to consist of a single convection cell in the vertical direction, which we therefore denote as mode 1. Equation (5.40) is independent of $\tilde{k}$ to $O\left(d^{2}\right)$, but numerical results confirm that $\tilde{k}=0$ is a local minimum of the neutral stability curve and so the wavelength of the most unstable mode is infinite. This proves to be the case for all values of $C<1 / 2$.

As the magnitude of $C$ is increased, the value of $R_{c}$ increases until when $C>1 / 2$, $\sigma$ becomes negative and the suspension unconditionally stable at small wavenumbers. 
This indicates that the instability occurs at larger wavenumbers when the sublayer concentration peak is below $z=-1 / 2$, as is confirmed by the numerical results given in the next section. When $C=0$ (so the cells accumulate at the top of the fluid layer) the problem reduces to one similar to that considered by CLS and HPK (in the case of no gyrotaxis), but our analysis appears to predict twice the critical Rayleigh number: 1440 as opposed to 720 . The variation arises from the minor difference in the definition of $d$ (see (4.10)) which includes the additional parameter $P . P$ quantifies the strength of the phototaxis in the suspension and thus affects the microorganisms' average upward swimming velocity, which varies throughout the fluid layer (see (3.8)) and is less than $V_{A}$ except at the lower surface. In previous models of bioconvection (CLS; HPK; Pedley et al. 1988 but not Pedley \& Kessler 1990), the average microorganism swimming velocity was assumed to be constant throughout the fluid layer. Equation (5.38) also indicates that increasing $\kappa$ increases $R_{c}$, which again we expect since this increases the magnitude of the horizontal diffusion that acts to damp the initial small perturbations.

\section{Stress-free upper surface}

We can perform a similar analysis for a stress-free upper surface, the only difference arising from the use of (5.11) for the boundary condition on $W$ at the upper surface. Examining the same balance as previously, the leading-order problem (5.22) and (5.23) has solution

$$
\Phi_{0}^{*}=-z, \quad W_{1}=-\frac{1}{48} \tilde{k}^{2} R_{0}\left(2 z^{4}+3 z^{3}-z\right),
$$

with $\sigma_{0}=0$. At the next order $\sigma_{1}=0$ and $\Phi_{1}$ has the same solution as previously (see (5.33)) so that along with the third-order boundary condition (5.36) we can now determine $\sigma_{2}$ using the solvability condition (5.35) which gives

$$
\sigma_{2}=\tilde{k}^{2}\left\{\frac{1}{720} R_{0}\left(1-\frac{9}{4} C\right)-\kappa\right\} .
$$

As we expect when $C=0$ the system is less stable to small perturbations, but the suspension is unconditionally stable at small wavenumbers for values of $C>4 / 9$ rather than $1 / 2$. A possible explanation for this behaviour is that the introduction of a stress-free upper surface allows the fluid motions from the unstably stratified region to penetrate more deeply into the buoyant fluid in the stable region. This increases the amount of energy required to maintain the unstable fluid motions so the initial stable state must possess more potential energy. As with the analysis for a rigid upper surface it is possible to proceed to higher orders, and again the critical Rayleigh number occurs at a vanishing wavenumber and is mode 1 .

\section{The numerical solution}

In this section we shall present numerical results to (5.13)-(5.14) for a complete range of values of $d$ and $C$. Neutral curves in the $(k, R)$-plane or growth rates $\sigma$ for a given value of $R$ were calculated using an eighth-order finite-difference scheme (NRK, provided by Dr D. R. Moore) based on Newton-Raphson-Kantorovich iteration (Cash \& Moore 1980) as described in HPK. The numerical scheme was rendered more efficient when $d \ll 1$ by using the scalings suggested by the asymptotic analysis in $\S 5.1$. For the deep layer $(d \gg 1)$, a suitable scaling is achieved by the transformation $\bar{z}=(z+C) d$ and $\bar{R}=R d^{-4}$ while keeping $W, \Phi^{*}=O(1)$. The numerical solutions were checked against the available asymptotic results for $R$ and shown to agree within the accuracy of the expansions. In addition, the numerical scheme was 

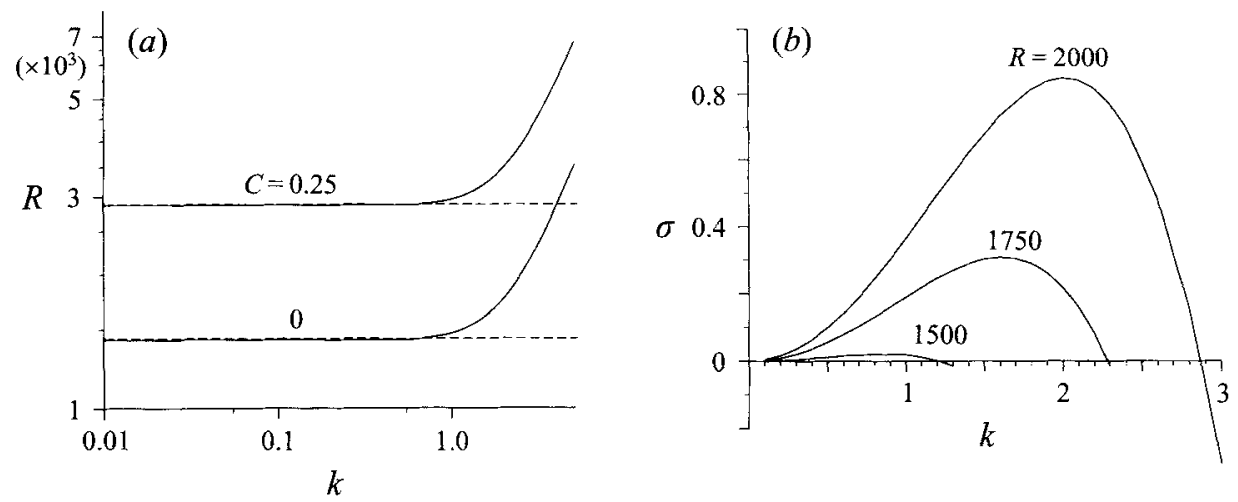

Figure 3. (a) Neutral curves for $d=0.1, C=0$ and 0.25 when the upper surface is rigid. The dashed lines are the asymptotic results. $(b)$ Growth rate, $\sigma$, plotted against wavenumber, $k$, for $d=0.1, C=0, S_{c}=20$ when the upper surface is rigid.

tested for many different parameter values and mesh sizes. Solutions for the same parameter values on different meshes or for the shallow and deep layer versions of the scheme always agreed to 5 (or more) significant figures for a minimum of 51 mesh points. Throughout this section we shall take $\kappa=1$.

\subsection{Stationary solutions}

\section{Rigid upper surface}

Neutral curves for various values of $d$ and $C$ are shown in figures 3-7. In figure 3(a) comparisons may be made between the numerical results and the shallow layer asymptotic results for $d=0.1$. As expected the numerical results confirm that the most unstable mode has a vanishing wavenumber. Equation (5.40) is only valid for values of $k \leqslant O(d)$ so that the solutions for $R^{(1)}(k)$ from the two methods were seen to diverge significantly for $k>1$. In the limit as $K \rightarrow 0$, the numerical and asymptotic results were found to agree to three significant figures for all values of $C<1 / 2$. For example, when $d=0.1$ and $C=0$ both methods give $R^{(1)} \rightarrow 1420$ as $K \rightarrow 0$, and when $C=0.25$ and $d=0.1$ both results yield $R^{(1)}(0)=2860$.

It is of course possible to obtain estimates of the expected wavelengths of the initial disturbances by plotting growth rate curves for values of the Rayleigh number above $R_{c}$. Growth rate curves for $C=0$ and $R=1500,1750$ and 2000 are illustrated in figure $3(b)$. Clearly once $R_{c}$ is exceeded in a shallow suspension the wavelengths of the resulting convective motions quickly become finite so that when $R=1500$, the maximum growth rate occurs at $k \simeq 0.9$ corresponding to a pattern wavelength of $7 \mathrm{~cm}$ for a fluid layer $1 \mathrm{~cm}$ deep. Increasing $R$ reduces pattern wavelength still further so that when $R=2000$ the maximum growth rate corresponds to a more realistic initial pattern wavelength of $3.1 \mathrm{~cm}$ for a layer $1 \mathrm{~cm}$ deep.

The effect of increasing $C$ when $d$ is small is shown in figure 4. As predicted by the asymptotic analysis, $R$ becomes asymptotically infinite as $k \rightarrow 0$ when $C>0.5$ and develops a minimum at wavenumbers of order 1 . Neutral curves for values of $d$ not covered by the asymptotic analysis in $\S 5.1$ are shown in figures 5-7. A selection of the results for critical Rayleigh number $\left(R_{c}\right)$ and wavenumber $\left(k_{c}\right)$ for different values of $d$ and $C$ are given in table 1 .

Particularly significant is the development of a minimum on $R^{(1)}(k)$ at non-zero values of $k$, for all values of $C$, as $d$ is increased. This contrasts with the results of CLS and HPK who reported that, for purely up-swimming algae, $k_{c}=0$ for all values 


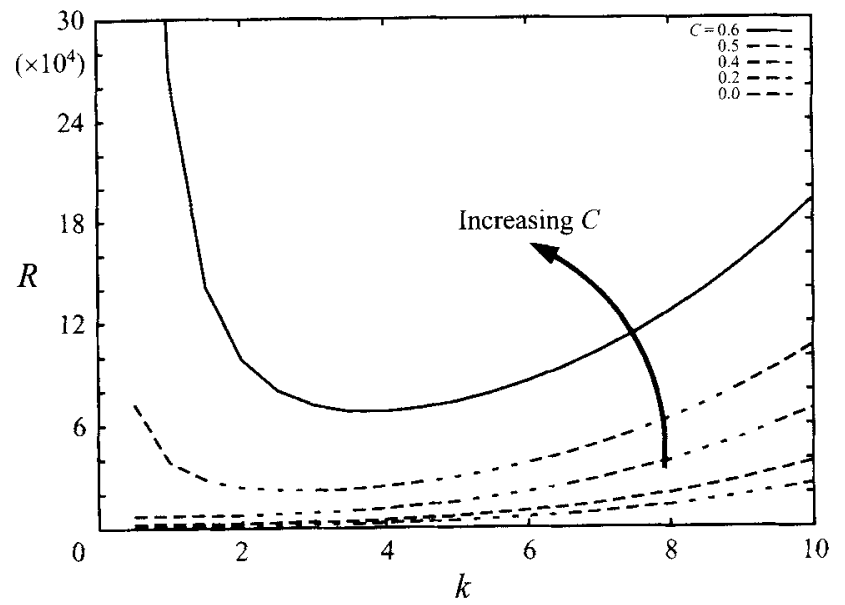

FIGURE 4. Neutral curves when $d=0.1$ showing the development of a non-zero critical wavenumber as $C$ is increased.

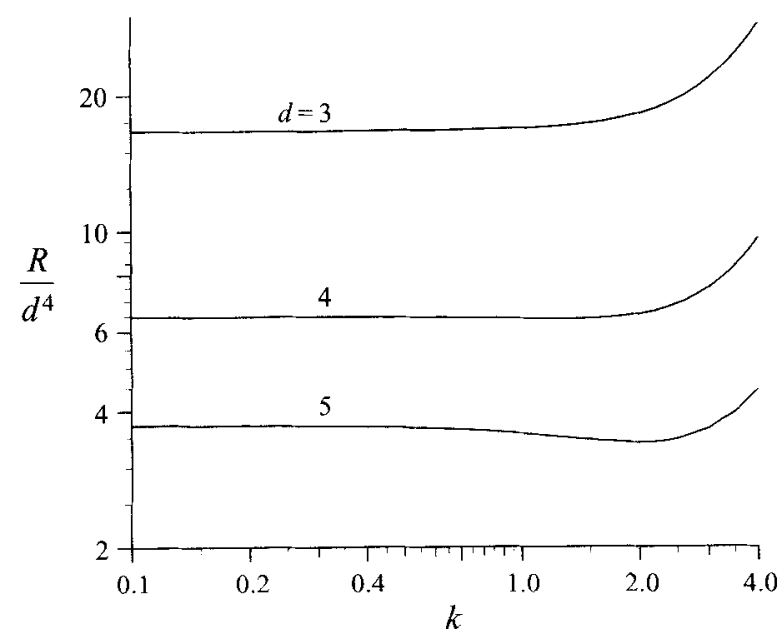

FIGURE 5. The development of the non-zero value for $k_{c}$ as $d$ is increased. $C=0$ and the upper surface is rigid.
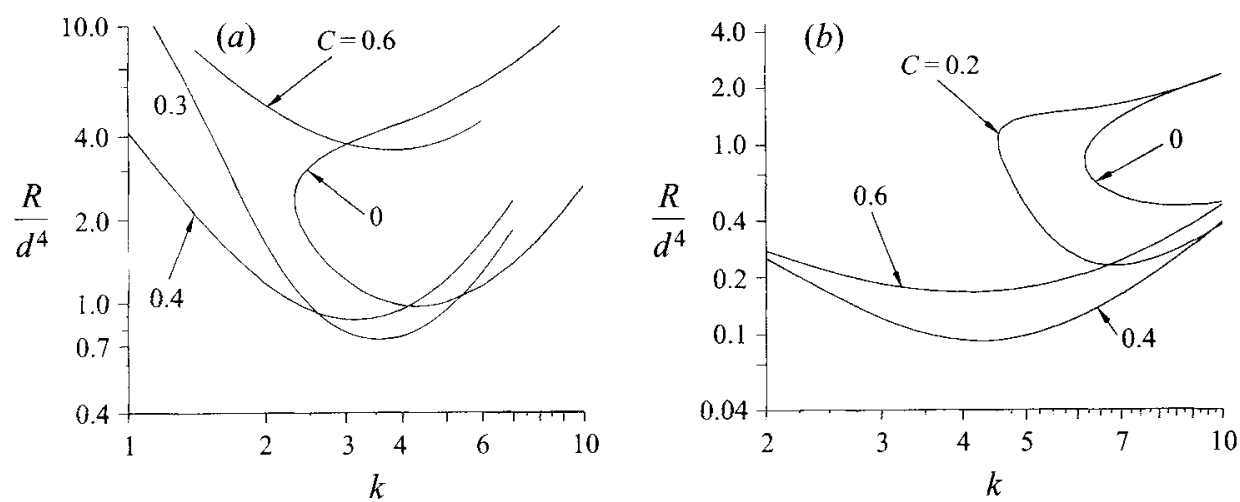

FIGURE 6. Neutral curves for $(a) d=10$ and $(b) d=20$ when the upper surface is rigid. 

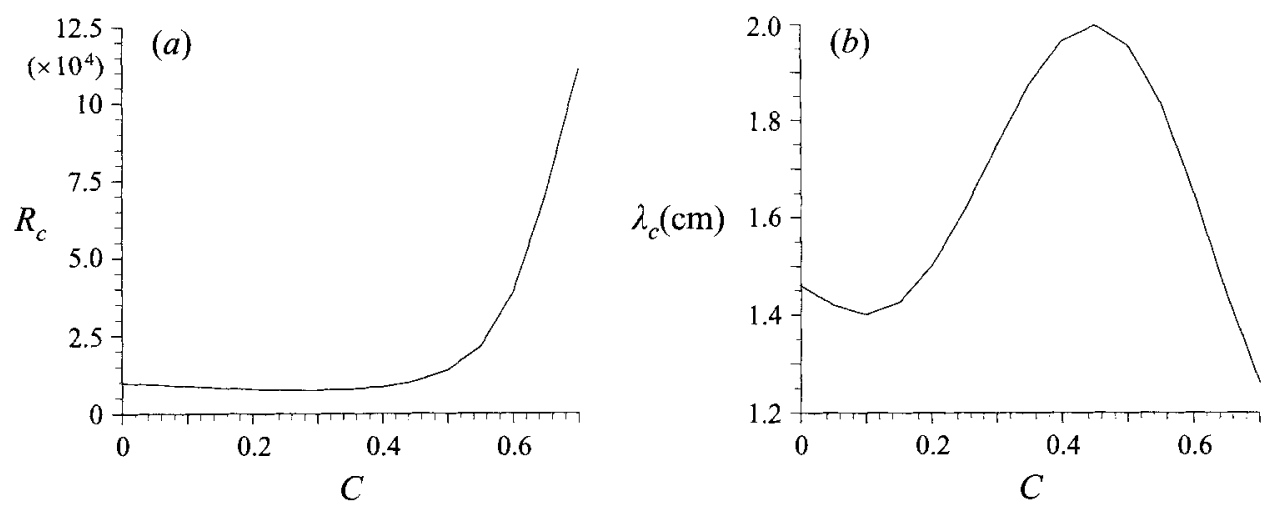

FIGURE 7. Variation of ( $a$ ) the critical Rayleigh number $R_{c}$ and $(b)$ the corresponding wavelength $\lambda_{c}$ (assuming $H=1 \mathrm{~cm}$ ) of the most unstable mode on the stationary branches for $d=10$ as $C$ is increased.

$\begin{array}{rlccc}d & C & \lambda_{c}(\mathrm{~cm}) & R_{c} & \text { Mode } \\ 4 & 0 & 5.24 & 1640 & 1 \\ 5 & 0 & 3.14 & 2120 & 1 \\ 5 & 0.1 & 3.30 & 2440 & 1 \\ 5 & 0.2 & 3.49 & 2960 & 1 \\ 10 & 0 & 1.46 & 9700 & 1 \\ 10 & 0.1 & 1.40^{*} & 8720^{*} & 1 \\ 10 & 0.3 & 1.75 & 7360 & 1 \\ 10 & 0.4 & 1.96 & 8690 & 1 \\ 10 & 0.6 & 1.65 & 35500 & 2 \\ 20 & 0 & 0.73 & 75300 & 1 \\ 20 & 0.1 & 0.73 & 59400 & 1 \\ 20 & 0.2 & 0.92^{*} & 36400^{*} & 1 \\ 20 & 0.3 & 1.16 & 21500 & 1 \\ 20 & 0.4 & 1.50 & 14700 & 1 \\ 20 & 0.6 & 1.53 & 26500 & 1\end{array}$

TABLE 1. Dependence of critical wavenumber and Rayleigh number on $d$ and $C$ for stationary solutions in a layer $1 \mathrm{~cm}$ deep with a rigid upper surface. A starred result indicates that a smaller minimum occurs on an oscillatory branch.

of $d$. Results for the case $C=0$ are illustrated in figure 5 using the deep layer scaling for the Rayleigh number, $R d^{-4}$. A non-zero critical wavenumber first occurs for $d \simeq 4$ for which $k_{c}=1.2$ and $R_{c}=1640$. For $d=3,4$ and 5, increasing the value of $C$, thus introducing a gravitationally stable layer at the top of the suspension, increases the critical Rayleigh number whereas $k_{c}$ remains relatively unchanged.

As $d$ is increased the value of $R_{c}$ increases. Neutral curves for $d=10$ and $d=20$ are drawn in figure 6. Here we observe that as $C$ is increased (see figure 7) $k_{c}$ varies with $C$ and $R_{c}$ initially decreases and then increases. Comparison of figures $6(a)$ and $6(b)$ shows also that the value of $C$ for which the suspension is most unstable increases as $d$ increases.

Such behaviour is not unusual in penetrative convection problems, see for example Matthews (1988), and Whitehead \& Chen (1970). Veronis (1963) reported that the introduction of a locally gravitationally stable region of fluid above an unstable region decreased the critical Rayleigh number and suggested that the addition of 
stable fluid at the top of the layer was offset by the relaxation of the upper no-slip boundary condition, allowing greater penetration into the stable region, so that the fluid motion could achieve an optimum form. Here, as the value of $C$ is increased, the relaxation of the upper no-slip boundary condition, which reduces viscous dissipation, is offset by the addition of stable fluid at the top of the layer, the buoyancy of which tends to inhibit convective fluid motions, so that eventually $R_{c}$ increases again, thus explaining the numerical results.

Another feature of the solutions is that, along certain parts of the neutral curves, the solution corresponding to the $R^{(1)}(k)$ branch is mode 2 not mode 1 . As $k$ is reduced below $k_{c}$ along such a branch, a single convection cell which extends throughout the whole layer is supplemented by a second smaller convection cell which forms at the bottom of the layer and grows in height as $k$ is decreased further. Where the branches loop back on themselves ( $d=10, C=0$ and $0.1, d=20, C=0$ and 0.2$)$ the upper part of the branch (which is smoothly connected to the lower branch) is mode 2 also. This behaviour occurred on all the branches illustrated in figure 6 except on the $R^{(1)}(k)$ branches for $d=10, C=0.3, C=0.4$ and $d=20, C=0.4$ and 0.6 . In the latter case, the mode 2 solution occurs for values of $k>k_{c}$, the second convection cell forming at the top of the layer increasing in size as $k$ is increased; whilst for $d=10$ and $C=0.6$ the whole of the branch is mode 2 , except for a region $k<k_{c}$ where the solution is mode 1 , so that in this instance the most unstable solution is actually mode 2 . HPK also reported the occurrence of mode 2 solutions on the $R^{(1)}(k)$ branches for some parameter ranges, although the the second convection cell formed at the top of the layer for values of $k<k_{c}$ and at the bottom of the layer for $k>k_{c}$.

When both the upper and lower boundaries are rigid, we may summarize the results as follows.

(a) For small values of $d$, the wavelength of the most unstable mode is infinite when $C<0.5$, and is finite for larger values of $C$.

(b) When $d$ is increased to $O(1)$ values, the wavelength of the most unstable mode becomes finite for all values of $C$, and increasing $C$ increases $R_{r}$.

(c) For large values of $d$, increasing $C$ from zero initially reduces $R_{c}$. In addition mode 2 solutions occur on parts of the $R^{(1)}(k)$ branches but the most unstable solution is always mode 1 except, if $C$ is greater than approximately 0.5 , then the most unstable solution can be mode 2 .

\section{Stress free upper surface}

To complete the results on stationary solutions, we now consider the situation where the upper boundary of the fluid is stress free. Results for this section are summarized in table 2 .

When $d$ is small the minimum on the $R^{(1)}(k)$ branch has a vanishing wavenumber $\left(k_{c}=0\right)$ and the numerical and asymptotic results agree to three significant figures when $d=0.1$.

Neutral curves for larger values of $d$ are shown in figures 8 and 9 and critical values for the wave and Rayleigh numbers are listed in table 2. There are some significant differences in behaviour between these results and those obtained when the upper boundary is rigid. Most notable is that the minimum of the $R^{1}(k)$ branch occurs at $k=0$ when $C=0$ for all the values of $d$ tested. This suggests that horizontal fluid shear at the upper surface is a significant factor in the formation of finite-wavelength patterns in this model.

Non-zero values of $C$ (thereby introducing a stable region above the unstable convective region) invariably result in non-zero values of $k_{c}$, provided that $d$ is large 


$\begin{array}{rlcrc}d & c & \lambda_{c}(\mathrm{~cm}) & R_{c} & \text { Mode } \\ 5 & 0 & \infty & 559 & 1 \\ 5 & 0.2 & \infty & 1100 & 1 \\ 10 & 0 & \infty & 1660 & 1 \\ 10 & 0.1 & 2.73 & 2912 & 1 \\ 10 & 0.2 & 2.62 & 3500 & 1 \\ 10 & 0.4 & 2.24 & 7370 & 1 \\ 10 & 0.6 & 1.65 & 35500 & 1 \\ 20 & 0 & \infty & 9310 & 1 \\ 20 & 0.01 & 1.75^{*} & 14600^{*} & 1 \\ 20 & 0.05 & 1.05^{*} & 24900^{*} & 2 \\ 20 & 0.1 & 0.98 & 28200 & 2 \\ 20 & 0.2 & 1.19 & 21000 & 1 \\ 20 & 0.4 & 1.85 & 11000 & 1 \\ 20 & 0.6 & 1.53 & 26500 & 1\end{array}$

TABLE 2. Dependence of critical wavenumber and Rayleigh number on $d$ and $C$ for stationary solutions in a layer $1 \mathrm{~cm}$ deep with a stress free upper surface. A starred result indicates that a smaller minimum occurs on an oscillatory branch.

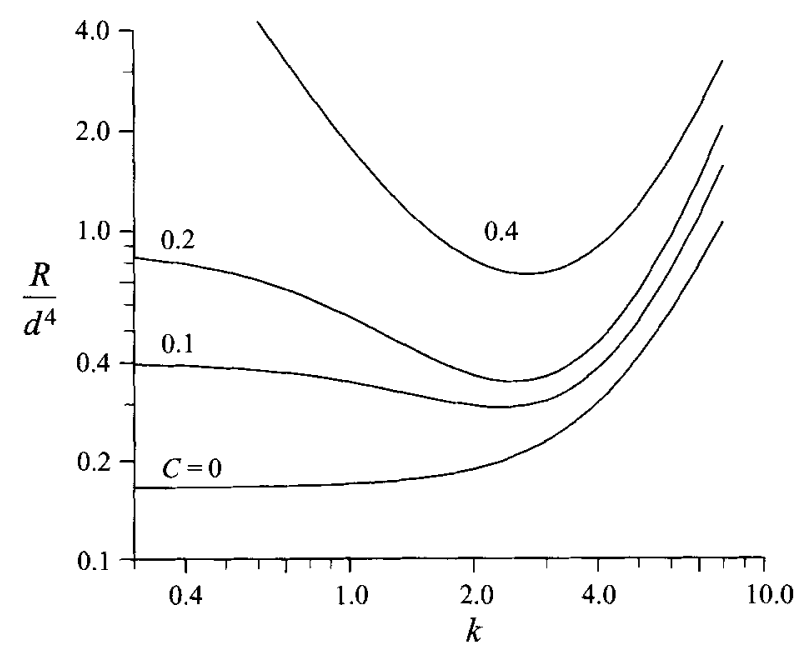

FiguRE 8. Neutral curves for $d=10$, stress-free upper surface.

enough. Another difference is that the increase in $R_{c}$ as $C$ is increased occurs for larger values of $d$ than for a rigid upper boundary. A good example of this behaviour is seen when $d=10$ (figure 8 ) where $R_{c}$ increases from approximately 1660 for $C=0$ to 7370 when $C=0.4$ (see table 2), unlike the rigid upper boundary case for $d=10$ where the Rayleigh number initially decreases and then increases with $C$ (see table 1). We might also anticipate that for large values of $C$, the differences that result from having a stress-free or rigid upper surface are minimized, so that the solutions to the eigenvalue problem should be similar. This indeed appears to be so as the results in tables 1 and 2 reveal, $R_{c}$ and $\lambda_{c}$ for the two different cases agreeing to three significant figures when $C=0.6$ for $d=10$ and $d=20$. For small values of $C$, tables 1 and 2 also reveal the large differences in the predicted wavelength of the initial convective motions, longer wavelengths occurring for a stress-free upper surface, particularly at $d=10$. On some of the neutral curves $(d=10, C=0,0.2$ and 0.3$)$ the solution 

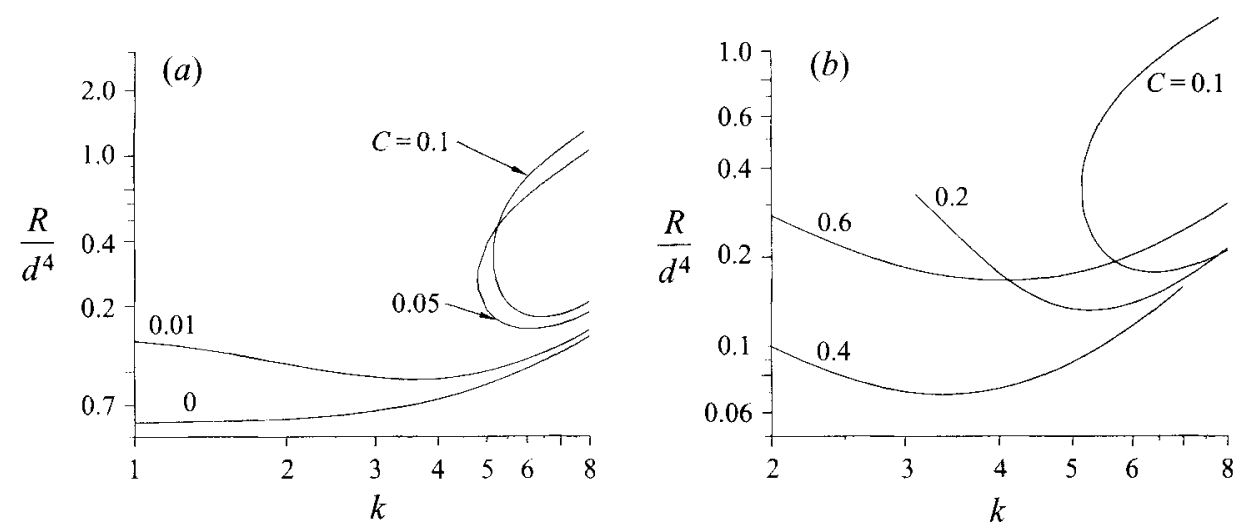

FIGURE 9. Neutral curves for $(a) d=20, C=0-0.1$ and $(b) d=20, C=0.1-0.6$ when the upper surface is stress free.

$\begin{array}{lll}\text { Cell radius } & a & 10^{-3} \mathrm{~cm} \\ \text { Cell volume } & v & 5 \times 10^{-10} \mathrm{~cm}^{3} \\ \text { Cell density ratio } & \Delta \rho / \rho & 5 \times 10^{-2} \\ \text { Cell diffusivity } & D_{H}=D_{V} & 5 \times 10^{-4} \mathrm{~cm}^{2} \mathrm{~s}^{-1} \\ \text { Swimming Speed } & V_{A} & 10^{-2} \mathrm{~cm}^{2} \mathrm{~s}^{-1} \\ \text { Mean concentration } & N_{0} & 10^{6} \mathrm{~cm}^{-3} \\ \text { Kinematic viscosity } & v & 10^{-2} \mathrm{~cm}^{2} \mathrm{~s}^{-1} \\ \text { Schmidt number } & S_{c} & 20 \\ \text { Sublayer depth } & l & 0.05 / P \mathrm{~cm}\end{array}$

TABLE 3. Estimates of typical parameters for a suspension of Chlamydomonas.

corresponding to the $R^{(1)}(k)$ branch switches smoothly from a mode 1 to mode 2 for some value of $k<k_{c}$ as a second convection cell forms at the bottom of the layer.

Neutral curves for $d=20$ are drawn in figure 9 and behaviour reminiscent of that seen in the previous section is obtained. Of the curves illustrated in figure $9(a)$ and $9(b)$, mode 2 solutions occur for values of $k<k_{c}$ on the $R^{(1)}(k)$ solution branch for $C=0.01,0.2$ and values of $k>k_{c}$ for $C=0.6$. In the latter case the second cell forms at the top of the layer, growing in depth as $k$ is increased. In two of the cases examined $(C=0.05$ and $C=0.1)$ the neutral curves loop back on themselves and solutions are mode 2 in an interval containing $k_{c}$. In these instances the most unstable stationary mode is mode 2 .

\subsection{Oscillatory solutions}

Overstable or oscillatory instabilities were found for both rigid and stress-free upper surfaces. A survey of values for the depth ratio $d(0.1,5,10$ and 20) and for the position of the cell concentration maximum in the steady state $C$, lead to the results detailed below. The Schmidt number $S_{c}$ was taken to be 20 throughout. Our aim thus far has been to illustrate general principles concerning the modelling of phototaxis in suspensions of algae, but to obtain estimates for many of the parameters required we shall now assume that we are dealing with a (non-gyrotactic) organism, otherwise similar to Chlamydomonas and use the same parameter values as HPK (see table 3). 


\begin{tabular}{|c|c|c|c|c|c|c|c|}
\hline & $d$ & C & $\lambda_{c}(\mathrm{~cm})$ & $R_{c}$ & $\operatorname{Im}\left(\sigma_{c}\right)$ & $T_{c}^{*}(\mathrm{~s})$ & Mode \\
\hline Rigid & $\{10$ & 0.1 & 0.98 & 8190 & 9.26 & 339 & 2 \\
\hline upper surface & $\{20$ & 0.2 & 1.96 & 11800 & 31.8 & 394 & 1 \\
\hline Stress-free & $\{20$ & 0.05 & 1.85 & 17100 & 29.4 & 427 & 2 \\
\hline upper surface & $\{20$ & 0.1 & 2.02 & 11900 & 31.5 & 398 & 2 \\
\hline
\end{tabular}

TABLE 4. Variation of critical wavenumber, Rayleigh number and period of oscillations with $d$ and $C$ for the most unstable overstable modes. Here $D=5 \times 10^{-4} \mathrm{~cm}^{2} \mathrm{~s}^{-1}, S_{\mathrm{c}}=20$ and we have arbitrarily chosen $P=1$ so that $l=0.05 \mathrm{~cm}$. Therefore when $d=20, H=1 \mathrm{~cm}$ and when $d=10$, $H=0.5 \mathrm{~cm}$.
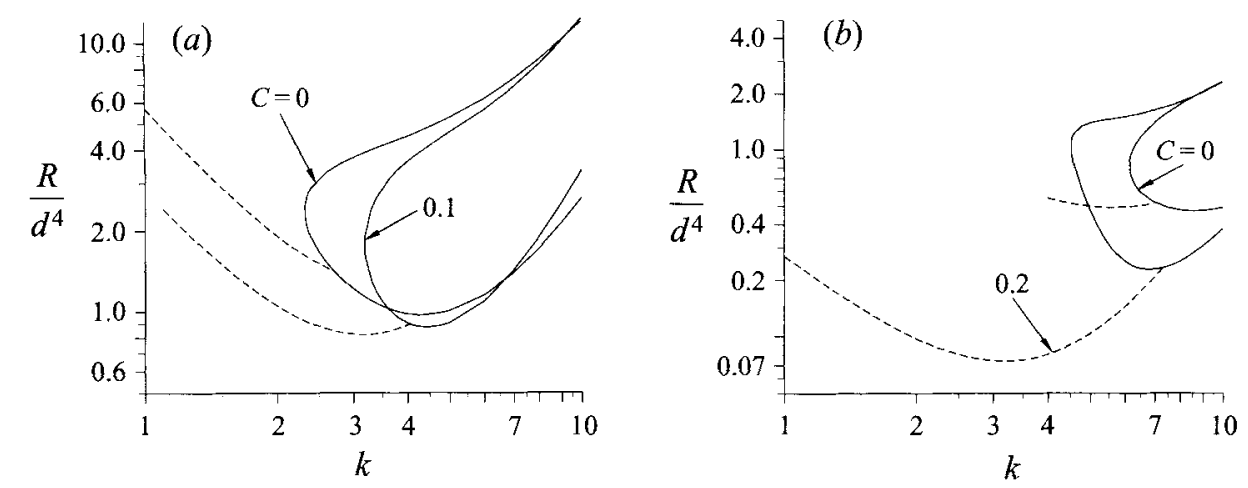

FIGURE 10. Stationary and oscillatory branches (dashed lines) ( $a$ ) for $d=10, C=0$ and 0.1 and $(b)$ for $d=20, C=0$ and 0.2 . In both cases $S_{c}=20$ and the upper surface is rigid.

The results from this section are summarized in table 4 .

\section{Rigid upper surface}

No oscillatory solutions were found for $d=0.1$ or 5 . Neutral curves for the cases $d=10$ and $d=20$ are shown in figure 10 and serve to illustrate the general behaviour observed for different values of $C$. Where overstability occurs, a single oscillatory branch bifurcates from the stationary branch at some wavenumber $k_{o}$ and defines a locus of points for $k \leqslant k_{o}$.

For example, when $d=10$ and $C=0$ the stationary branch loops back on itself. The oscillatory solution bifurcates from it at $k=2.7$, but in this particular case the stationary solution is still the most unstable. When $C=0.1$ the oscillatory branch develops a smaller minimum than that found on the stationary branch so in this instance $\left(k_{c}, R_{c}\right)=(3.2,8190)$ lies on the oscillatory branch and the solution is mode 2 , the lower convection cell occupying the bottom one third of the layer. $\operatorname{Im}(\sigma)$ has a value of 9.26 so that the period for overstability is $339 \mathrm{~s}$. When $C$ is increased to 0.4 the behaviour is similar to that obtained for $C=0.1$, the stationary branch once again possessing the most unstable solution, but when $C=0.6$ no oscillatory behaviour was found.

When $d=20$ the pattern of behaviour seen for $d=10$ is repeated (figure $10 b$ ) but there is a greater tendency for the most unstable mode to be oscillatory. When $C=0.2$, the minimum on the oscillatory branch is significantly smaller than that on the stationary branch giving $\left(k_{c}, R_{c}\right)=(3.2,11800)$. Here the solution is mode 1 and 


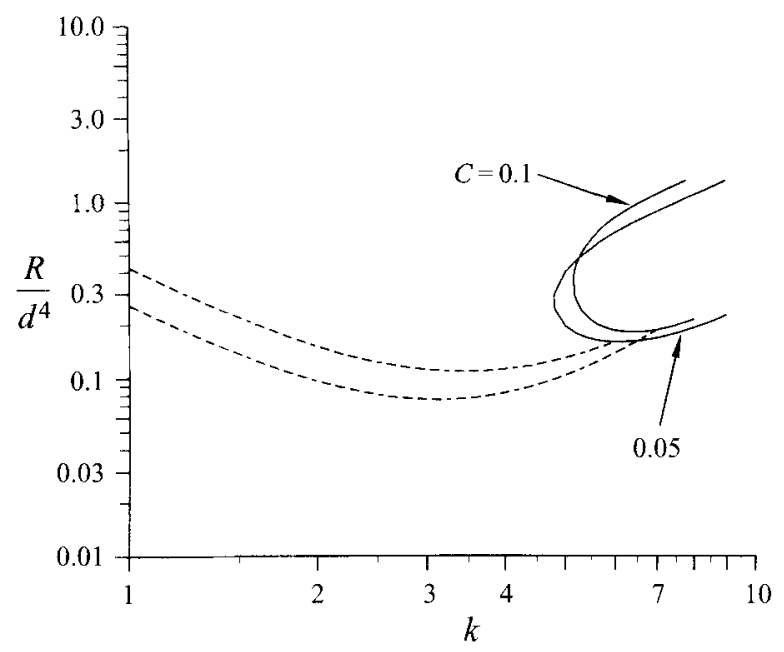

FIGURE 11. Stationary and oscillatory branches (dashed lines) for $d=20, C=0.05$ and $0.1 . S_{c}=20$ and the upper surface is stress free.

$\operatorname{Im}(\sigma)=31.8$ so the period of the resulting oscillations is $395 \mathrm{~s}$. Larger values of $C$ (e.g. $C=0.3, C=0.4$ and $C=0.6$ ) yielded no oscillatory solutions.

Oscillatory branches were always found when the stationary neutral curves looped back, but the absence of this behaviour did not preclude the existence of oscillatory solutions.

\section{Stress-free upper surface}

Oscillatory behaviour was also found when the upper surface was stress free, although apparently for a smaller range of parameter values than was the case when the upper surface is rigid. No oscillatory solutions were found when $C=0$ or when $d=0.1,5$ or $d=10$. When $d=20$ the stationary branches for $C=0.05$ and $C=0.1$ loop back and oscillatory branches were found (see figure 11). None of the other values of $C$ examined, for which stationary branches are illustrated in figures $8-9$, revealed the existence of overstable modes. Both the oscillatory branches develop substantially smaller minima than those found on the stationary branches, so that the initially most unstable modes are oscillatory in nature. For $C=0.05$, $\left(k_{c}, R_{c}\right)=(3.4,17100)$ and $\operatorname{Im}(\sigma)=29.4$ which gives a period for overstability of $427 \mathrm{~s}$. When $C=0.1,\left(k_{c}, R_{c}\right)=(3.1,11900)$ and $\operatorname{Im}(\sigma)=31.5$ so that the period is $398 \mathrm{~s}$.

\section{The mechanism for overstability}

The fundamental difference between this model for phototaxis and that of CLS for pure up swimming, for which the principle of exchange of stabilities can be proved, is that the average swimming velocity is variable (see (2.1) and (3.8)) and therein must lie the mechanism for overstability.

To understand the mechanism, we consider an example of oscillatory convection when $d=20$ and $C=0.2$. In the equilibrium state, the maximum concentration of cells is at $z=-0.2$ near the top of the layer, there is no flow, and there is a balance between the vertical flux due to phototaxis and diffusion down the cell concentration gradient. Suppose that the small perturbation to this basic state is in the form of a two-dimensional roll pattern with a planform $f(x, y)=\cos k x$ (see (5.6)), and then figure $12(a)$ for $W(z)$ illustrates the flow at $x=0$ at a particular instant of time. 

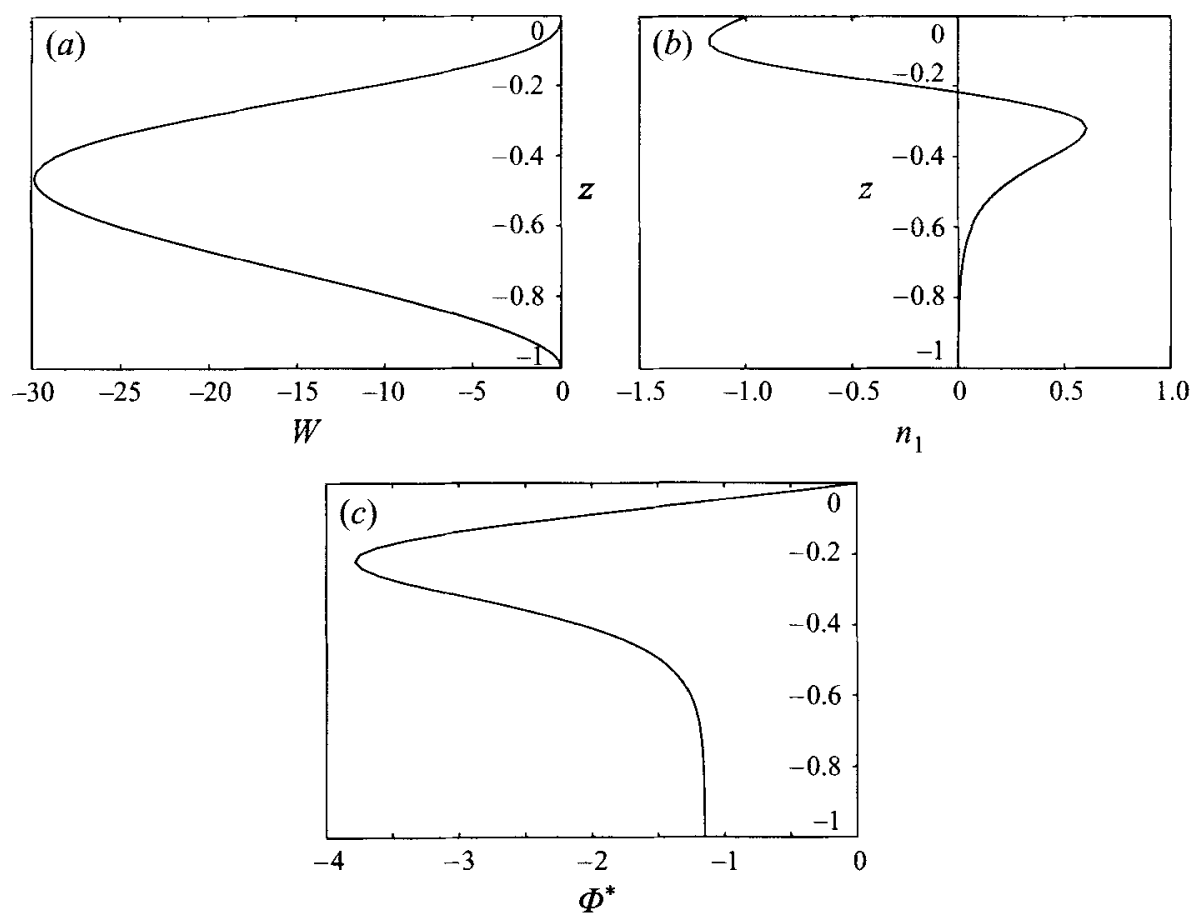

FIGURE 12. Profiles of $(a)$ the vertical velocity $W(z),(b)$ the perturbation to the cell concentration $n_{1}(z)$ and $(c)$ the perturbation to the shading $\Phi^{*}(z)$ for an oscillatory solution when $d=20, C=0.2$, $R=11800, k=3.2$ and $S_{c}=31.8$. Plot $(b)$ shows that the upper region $(z>-0.2)$ is buoyant and opposes the downwelling. The mean vertical swimming velocity is proportional to the shading and contributes a non-uniform flux of cells which tends to further deplete the upper region and reverse the flow.

$W(z)$ is negative throughout the whole layer corresponding to a mode 1 instability with downwelling at $x=0$.

In figure $12(b)$ we show $n_{1}(z)$, the perturbation to the cell concentration, at $x=0$ and at the same instant of time. $n_{1}(z)$ is negative for $z>-0.2$ so there is a region of positive buoyancy which opposes and is out of phase with the downwelling. There is also a lower region where $n_{1}(z)$ is positive, and thus negatively buoyant, which promotes downflow, but the upper region is dominant for the parameter values in this example.

At $x=0$ and the same instant, $\Phi^{*}(z)$, as defined in (5.12), is proportional to $\int_{z}^{0} n_{1}(s) \mathrm{d} s$ and thus may be thought of as the perturbation to the shading. It is plotted in figure $12(c)$. It is negative throughout the layer, implying less shading at any particular depth than in the equilibrium state and it has a marked minimum at $z \approx-0.2$ that is a common feature of oscillatory bioconvection. From (3.8), it can be seen that the perturbation to the mean vertical swimming velocity due to phototaxis is proportional to $\Phi^{*}(z)$, and the position of the minimum results in a non-uniform perturbation flux of cells away from the upper region enhancing the positive buoyancy and acting to oppose the downflow. It is this non-uniform contribution to the flux due to shading that provides a stabilizing mechanism to compete with the RayleighTaylor overturning instability and leads to the oscillatory modes of convection for suitable parameter values when phototaxis is relatively important $(P \gg 1)$ and thus $d \gg 1$. 
Oscillatory patterns have not, as yet, been observed in suspensions of algae. In any case it is unlikely that it would be possible to observe the type of oscillatory behaviour we have analysed above because the convective motions become fully nonlinear on a timescale substantially less then the predicted period for overstability.

\section{Discussion}

In this paper we have introduced a theoretical framework which allows the linear stability of a suspension of negatively buoyant, phototactic, motile microorganisms, subject to uniform illumination from above, to be analysed for the first time.

Previous studies of the stability of a layer of microorganisms of finite depth have considered a suspension of cells that swim upward at a constant speed (CLS) and a suspension of up-swimming gyrotactic cells (HPK). In both models the up swimming in the layer leads to a density stratification with the denser fluid on top, which triggers the resulting convective motions. CLS found that the wavelength of the most unstable mode was always infinite and were able to show that the instability was always non-oscillatory. HPK found that, when gyrotaxis was incorporated with up swimming, the wavelength of the most unstable mode was finite provided that the upper surface was rigid, and furthermore that oscillatory instabilities occurred in certain parameter ranges.

This model differs from predecessors in three important respects. Firstly there is no gyrotaxis. Secondly, the algal cells may swim upward toward the light source (positive phototaxis), or downward away from the light source (negative phototaxis), their reactions being controlled by the flux of light reaching their photoreceptors, which in turn is a function of cell concentration (see (3.8)). Thirdly, the equilibrium concentration profile can form at any position in the layer, depending on the intensity of the light source, with the result that, in general, a stable layer of fluid overlies a gravitationally unstable layer.

The results of the linear stability analyses in $\$ \$ 5.1$ and 6 indicate that the initial pattern spacing depends on $d$ and $C$. In particular for small values of $d$, the most unstable mode has a vanishing wavenumber whilst for larger values it is non-zero. For any given value of $C$, the suspension becomes more unstable as the depth of the layer is increased. For large values of $d$, the introduction of a small stable region at the top of the layer also makes the suspension more unstable. More surprising is the existence of oscillatory modes that occur for certain ranges of $C$ when $d$ is large and are driven by a non-uniform flux of cells due to the perturbations in shading. This results in a positive feedback mechanism that promotes the growth of positively and negatively buoyant regions in the suspension. In all the cases studied when a gravitationally stable region occurs at the top of the layer, the fluid motions in the unstable layer penetrate the stable region totally so that convective motions occur throughout the depth of the layer. A significant difference between the results for rigid and stress-free upper surfaces occurs when $C=0$. In the former case the most unstable mode has a non-zero wavenumber (provided $d>4$ ), whereas when the upper surface is stress free the most unstable mode has a zero wavenumber for all values of $d$. HPK found similar behaviour for a suspension of gyrotactic cells.

If the theory developed in this paper is to be applied then it is important to determine whether or not the basic equilibrium state ever forms. HPK and Pedley et al. (1988) addressed this problem by considering whether sufficient time had elapsed before patterns were seen for the residual fluid motions caused by mixing to have decayed and for the equilibrium profile to have been established. Pedley et al. (1988) 
estimated the decay time for the turbulent eddies as $H / U$, where $H$ is the depth and $U$ the typical stirring velocity. HPK supposed that the fluid and container were initially in solid-body rotation with angular velocity $\Omega$ and that at $t=0$ the container was brought to rest. Using Ekman boundary layer theory they were able to estimate the timescale for the decay of the swirling fluid motions. In the example they gave, if $\Omega=1 \mathrm{~s}^{-1}$ the decay time is $\approx 10 \mathrm{~s}$, which is larger then $H / U$, if $U>0.1 \mathrm{~cm} \mathrm{~s}^{-1}$ when $H=1 \mathrm{~cm}$.

The time taken for the equilibrium profile to form is on the order of the time taken for a typical cell to swim to $z^{*}=-C H$. This is difficult to estimate since cell swimming speed is a function of cell concentration, but if we consider the motion of a single cell in a uniform suspension $\left(n^{*}\left(z^{*}\right)=N_{0}\right)$ then cell swimming speed $V$ from (2.1) and (3.8) is

$$
V=-V_{A} P\left(\frac{z^{*}}{H}+C\right) .
$$

Taking $P=1, H=1 \mathrm{~cm}, V_{A}=100 \mu \mathrm{m} \mathrm{s}^{-1}$ and $C=0$, the average swimming speed of the cell when it swims from the bottom to the top of the layer is $50 \mu \mathrm{m} \mathrm{s}^{-1}$, so we estimate that the timescale to traverse the layer is on the order of $200 \mathrm{~s}$. Alternatively if $C=0.5$, the organism has to swim a smaller vertical distance to reach optimum illumination conditions, but its average speed is smaller at $25 \mu \mathrm{m} \mathrm{s}^{-1}$ which again gives the estimate of $200 \mathrm{~s}$ for the equilibrium state to form.

Formation of the equilibrium state given in (4.9) requires that the suspension is initially absolutely uniform. Any small non-uniformities in cell concentration (caused by stirring for example) will cause horizontal variations in the illumination with the result that the position of the concentrated sublayer will vary horizontally too. Therefore under experimental conditions it will be difficult to obtain the equilibrium state across the whole layer unless the suspension is initially very well mixed.

A major limitation of the analysis we have presented is the assumption that the absorption of light across the suspension is weak $\left(0<\alpha^{*} n^{*} H \ll 1\right)$ so that the light intensity throughout the suspension is always in the linear range close to $I_{c}$. This assumption allowed us to derive a simplified expression for the average cell swimming direction $((2.1)$ and (3.8)) that produced a cell conservation equation to which there was an analytic solution. For the purposes of the linear stability analysis, i.e. during the formation of the sublayer and the initial stages of the resulting instability, the assumption that the absorption is weak is probably justified provided that the cell concentration is initially uniform, but its validity must be questioned once the concentrated plumes or sheets of falling cells characteristic of bioconvection have fully developed. Within these structures there will be substantial shading so that algal cells will experience light intensities ranging from $I_{s}$, at the top of a plume close to the fluid surface, to almost zero at the bottom of a plume. Therefore a fully non-linear investigation of phototactic bioconvection should involve using a form of the taxis function $T(I)$ that covers the full range of possible values of $I$ (see (2.3)) and the general exponential expression for the Lambert-Beer law.

The work presented in this paper should include a comparison of the theoretical predictions with quantitative experimental studies of bioconvection in suspensions of phototactic algae. Unfortunately no such data as yet exist. Besides the difficulties in obtaining the equilibrium state mentioned above and measuring parameters such as $l$, $D_{V}, D_{H}$ and $C$, the study of bioconvection in suspensions of phototactic algae presents some additional problems. The first involves identifying a suitable species of algae that only displays phototactic responses. Most species of algae, besides displaying various 
types of photoresponse, are in addition geotactic or gyrotactic so that that their swimming trajectories represent a balance between different environmental effects (Häder 1987b; Kessler, Hill \& Häder 1992). Assuming that it is possible to identify a suitable candidate species of microorganism, it is then necessary to perform additional experiments to determine $I_{c}$ and the light transmittance of a suspension of cells so that $P$ can be calculated.

Measurements of bioconvection patterns in different suspensions of algae $1 \mathrm{~cm}$ deep suggest typical initial spacings vary between 1 and $3 \mathrm{~cm}$. The predicted initial wavelengths for $C=0$ in table 4.1 appear to agree moderately well with this observation. The mean cell concentration $N_{0}$ is related to $R$ by (5.17) so that using the parameters in table 4.3 for Chlamydomonas and setting $P=1$ and $H=1 \mathrm{~cm}$ gives $R=R_{c}$ when $N_{0}=N_{0 c}=7.5 \times 10^{5}$ cells $\mathrm{cm}^{-3}$ which again seems to be reasonably close to typical experimental data. Direct comparison between these results and those of HPK is difficult but in general, when $C=0$, the wavelength of the most unstable mode is shorter for a suspension of phototactic algae than a suspension of gyrotactic algae. In the case detailed above for a (non-gyrotactic) species of Chlamydomonas, $\lambda_{c}=0.73 \mathrm{~cm}$ (when $H=1 \mathrm{~cm}$ ) whereas, using their best guess for the strength of gyrotaxis in Chlamydomonas, HPK obtained critical wavelengths varying between 0.86 and $7 \mathrm{~cm}$ depending on the eccentricity of the microorganisms' ellipsoidal body shape.

Finally, we note that the model for phototaxis and shading is very general and could be applied to a number of other interesting problems involving populations of phototactic microorganisms, for example investigating the effect that lateral (or side) illumination has on bioconvection patterns, or modelling the phototactic behaviour of populations of algae in a porous media (e.g. snow or sand) that absorbs and scatters light.

We are grateful for many helpful discussions with Professors J. O. Kessler and T. J. Pedley and to the reviewers for several corrections and improvements. R.V.V. was supported by an EPSRC Earmarked Studentship in Mathematical Biology.

\section{Appendix. Solution of the cell conservation equation}

We wish to solve the equation

$$
\frac{\mathrm{d} n^{*}}{\mathrm{~d} z^{*}}-d n^{*}\left(\int_{z^{*}}^{0} n^{*} \mathrm{~d} z^{\prime}-C H N_{0}\right)=0 \text { where } d=\frac{V_{A} A I_{c} \alpha^{*}}{D_{V}} .
$$

Rearranging this equation and differentiating with respect to $z^{*}$ to eliminate the integral gives the second-order, non-linear ODE

$$
n^{*} \frac{\mathrm{d}^{2} n^{*}}{\mathrm{~d} z^{* 2}}-\left(\frac{\mathrm{d} n^{*}}{\mathrm{~d} z^{*}}\right)^{2}+d n^{* 3}=0 .
$$

Fortunately this equation can be reduced to a linear first-order ODE using a pair of transformations (see Kamke 1967, p. 573). If we write

$$
p\left(n^{*}\right)=\frac{\mathrm{d} n^{*}}{\mathrm{~d} z^{*}}
$$

then (A 2) is reduced to the Bernoulli equation

$$
n^{*} p \frac{\mathrm{d} p}{\mathrm{~d} n^{*}}-p^{2}+d n^{* 3}=0 .
$$


Then, using the transformation

$$
p\left(n^{*}\right)=n^{* 3 / 2} u(t), \quad t=\ln n^{*}
$$

equation (A 4) may be further simplified to

$$
u \frac{\mathrm{d} u}{\mathrm{~d} t}+\frac{1}{2} u^{2}+d=0
$$

which is separable, and may be solved using elementary methods to give the general solution

$$
n^{*}\left(z^{*}\right)=\frac{K^{2}}{2 d} \operatorname{sech}^{2}\left(\frac{1}{2} K\left(z^{*}+L\right)\right)
$$

where $K$ and $L$ are constants of integration. The value of $L$ lies between 0 and $-H$ and depends on the position in the suspension where the light intensity takes the value $I_{c}$ (i.e. at $z^{*}=-C H$ ). Thus, provided that the initial cell concentration profile is precisely uniform, the vertical position where the light intensity is equal to $I_{c}$ remains the same, and $L=C H$.

\section{REFERENCES}

CASH, J. R. \& MOORE, D. R. 1980 A high order method for the numerical solution of two point boundary value problems. BIT 20, 44-52.

Chandrasekhar, S. 1961 Hydrodynamic and Hydromagnetic Stability. Oxford University Press.

Childress, S., Levandowsky, M. \& Spiegel, E. A. 1975 Pattern formation in a suspension of swimming microorganisms: equations and stability theory. J. Fluid Mech. 63, 591-613 (referred to herein as CLS).

Childress, S. \& PeYret, R. 1976 A numerical study of two-dimensional convection patterns. J. Méc. 15, 753-779.

Colombetti, G. \& Petracchi, D. 1989 Photoresponse mechanisms in flagellated algae. Crit. Rev. Plant Sci. 8, 309-355.

DuYsENS, L. N. M. 1956 The flattening of the absorption spectrum of suspensions, as compared to that of solutions. Biochim. Biophys. Acta 19, 1-12.

Foster, K. W. \& Sмyтt, R. D. 1980 Light antennas in phototactic algae. Microbiol. Rev. 40, $572-630$.

HäDER, D.-P. 1987 a Photomovement in eukaryotic microorganisms. Photochem. Photobiophys. Suppl. 203-214.

HÄDER, D.-P. $1987 b$ Polarotaxis, gravitaxis, and vertical phototaxis in the green flagellate, Euglena gracilis. Arch. Mikrobiol. 147, 179-183.

Herdan, G. 1960 Small Particle Statistics, 2nd Edn. Butterworth \& Co.

HiLl, N. A. \& HÄDER, D.-P. 1996 A biased random walk model for the trajectories of swimming micro-organisms. Submitted to J. Theor. Biol.

Hill, N. A., Pedley, T. J. \& Kessler, J. O. 1989 Growth of bioconvection patterns in a suspension of gyrotactic micro-organisms in a layer of finite depth. J. Fluid Mech. 208, 509-543 (referred to herein as HPK).

Hillesdon, A. J. 1994 Pattern formation in a suspension of swimming bacteria. PhD thesis, University of Leeds.

Hillesdon, A. J., Pedley, T. J. \& Kessler, J. O. 1995 The development of concentration gradients in a suspension of chemotactic bacteria. Bull. Math. Biol. 57, 299-344.

KAMKE, E. 1967 Differentialgleichungen Lösungsmethoden und Lösungen, Vol 1. Akademische Verlagsgesellschaft Geest \& Portig K.-G.

KessLER, J. O. 1985 Co-operative and concentrative phenomena of swimming microorganisms. Contemp. Phys. 26, 147-166.

KessLer, J. O. 1986 The external dynamics of swimming microorganisms. In Progress in Phycological Research, vol. 4 (ed. F. E. Round \& D. J. Chapman). Bristol Biopress Ltd. 
Kessler, J. O. 1989 Path and pattern - the mutual dynamics of swimming cells and their environment. Comments on Theor. Biol. 212, 85-108.

Kessler, J. O., Hill, N. A. \& HäDER, D.-P. 1992 Orientation of swimming flagellates by simultaneously acting external factors. J. Phycology 28, 816-822.

LOEFFER, J. B. \& MEFFERD, R. B. 1952 Concerning pattern formation by free swimming microorganisms. The American Naturalist 86, 325-329.

MatThews, P. C. 1988 A model for the onset of penetrative convection. J. Fluid Mech. 188, 571-583.

Pedley, T. J., Hill, N. A. \& Kessler, J. O. 1988 The growth of bioconvection patterns in a uniform suspension of gyrotactic micro-organisms. J. Fluid Mech. 195, 223-237.

Pedley, T. J. \& Kessler, J. O. 1990 A new continuum model for suspensions of gyrotactic micro-organisms. J. Fluid Mech. 212, 155-182.

Pedley, T. J. \& KessleR, J. O. 1992 Hydrodynamic phenomena in suspensions of swimming microorganisms. Ann. Rev. Fluid Mech. 24, 315-358.

PlatT, J. R. 1961 Bioconvection patterns in cultures of free swimming algae. Science 133, 1766-1767.

Roennberg, T., COlfaX, G. N. \& Hastings, J. W. 1989 A circadian rhythm of population behaviour in Gonyaulax polyedra. J. Biol. Rhythms 4, 201-216.

Straughan, B. 1993 Mathematical Aspects of Penetrative Convection. Longman Scientific \& Technical.

Veronis, G. 1963 Penetrative convection. Astrophys. J. 137, 641-663.

VINCENT, R. V. 1995 Mathematical modelling of phototaxis in motile microorganisms. PhD thesis, University of Leeds.

WAGER, H. 1911 On the effect of gravity upon the movements and aggregation of Euglena viridis. Ehrb., and other microorganisms. Phil. Trans. R. Soc. Lond. B 201, 333-390.

WhitehEAD, J. A. \& CHEN, M. M. 1970 Thermal instability and convection of a thin fluid layer bounded by a stably stratified region. J. Fluid Mech. 40, 549-576. 
